\title{
Confined Growth of ZIF-8 Nanocrystals with Tunable Structural Colors
}

\author{
Chang, Bingdong; Yang, Yuanqing; Jansen, Henri; Ding, Fei; Mølhave, Kristian; Sun, Hongyu
}

Published in:

Advanced Materials Interfaces

Link to article, DOI:

10.1002/admi.201701270

Publication date:

2018

Document Version

Peer reviewed version

Link back to DTU Orbit

Citation (APA):

Chang, B., Yang, Y., Jansen, H., Ding, F., Mølhave, K., \& Sun, H. (2018). Confined Growth of ZIF-8 Nanocrystals with Tunable Structural Colors. Advanced Materials Interfaces, 5(9), [1701270]. https://doi.org/10.1002/admi.201701270

\section{General rights}

Copyright and moral rights for the publications made accessible in the public portal are retained by the authors and/or other copyright owners and it is a condition of accessing publications that users recognise and abide by the legal requirements associated with these rights.

- Users may download and print one copy of any publication from the public portal for the purpose of private study or research.

- You may not further distribute the material or use it for any profit-making activity or commercial gain

- You may freely distribute the URL identifying the publication in the public portal 
DOI: 10.1002/ ((please add manuscript number))

Full Paper

\section{Confined growth of ZIF-8 nanocrystals with tunable structural colors}

Bingdong Chang* , Yuanqing Yang* , Henri Jansen , Fei Ding , Kristian Mølhave and Hongyu Sun *

B. D. Chang, Prof. H. Jansen

DTU Danchip, Technical University of Denmark, Kongens Lyngby 2800, Denmark

Dr. Y. Q. Yang, Dr. F. Ding

SDU Nano Optics, University of Southern Denmark, Campusvej 55, DK-5230 Odense M, Denmark

Prof. K. Mølhave, Dr. H. Y. Sun

Department of Micro- and Nanotechnology, Technical University of Denmark, Kongens

Lyngby 2800, Denmark

E-mail: hsun@nanotech.dtu.dk

Keywords: ZIF-8; gold nanoparticles; atomic layer deposition; structural colors

Abstract:

Zeolitic imidazolate frameworks (ZIF-8) have promising applications as sensors or catalysts due to their highly porous crystalline structures. While most of the previous studies are based on ZIF-8 crystals either in isolated particles in aqueous environments or in a compact colloidal form, here we report a facile route to achieve wafer-based isolated ZIF-8 nanocrystals. The fabrication includes the growth of compact zinc oxide film by atomic layer deposition, the dispersion of gold nanoparticles as inhibitors, and followed by crystallization transformation. By adjusting the concentration of gold nanoparticles, the density of ZIF-8 nanocrystals can be controlled and the sizes of individual ZIF-8 crystals can be scaled down to $\sim 100 \mathrm{~nm}$. We also observe a wide range of structural colors generated by the ZIF-8 nanocrystals, which can be attributed to the size-dependent resonant scattering as verified by finite-difference time-domain simulations and classical Mie theory. The scalable fabrication of wafer-based ZIF-8 nanocrystals empowered with tunable optical properties paves a new way to explore the promising applications in nanophotonics and bionanotechnology. 


\section{Introduction}

Metal-organic frameworks (MOFs) have been widely studied in recent years due to their unique structures and applications in environment and energy. As a typical MOF, zeolitic imidazolate framework-8 (ZIF-8) possesses three dimensional zeolite crystal structure which is constructed by bridging zinc ions with methylimidazolate (MeIm) ligands. Compared with other ZIF structures, ZIF-8 crystals show large cavity size (11.6 ̊), small connecting apertures (3.4 $\AA$ ), and high thermal/chemical stability, endowing them outperform other MOF structures in a wide range of applications, including heterocatalysis, ${ }^{[1,2]}$ low- $\kappa$ dielectrics microelectronic devices ${ }^{[3]}$ and gas adsorption. ${ }^{[4]}$

The crystallization of ZIF-8 requires both zinc ion sources and imidazolate linkers. Traditional bulk synthesis methods have been well studied and established. ${ }^{[5-9]}$ In the synthesis, soluble zinc salts $\left(\mathrm{Zn}\left(\mathrm{NO}_{3}\right)_{2}, \mathrm{ZnSO}_{4}, \mathrm{ZnCl}_{2}\right.$, etc) are used to provide zinc ions. Nevertheless, the yielded powder-like ZIF-8 crystals are difficult to be integrated into microor nano- electro-mechanical systems (MEMS or NEMS), where a mechanically robust structure is preferred. Recent studies show that solid-state $\mathrm{ZnO}$ nanostructures (nanoparticles, nanowires, or nanofilms) can also be used as zinc ion sources to direct the growth of ZIF-8 crystals, which inherit the initial morphology of ZnO nanostructures. For example, polycrystal ZIF-8 thin film can be fabricated by converting ZnO layer grown either by atomic layer deposition (ALD) or sputtering. ${ }^{[10]}$ By combining metal oxide vapor deposition and consecutive vapor solid reaction, ZIF-8 thin films with high quality can be deposited directly. ${ }^{[11]}$ Based on the compact ZIF-8 films, traditional top-down micro- and nanofabrication techniques (such as standard photolithography ${ }^{[12]}$ and nanoimprint lithography ${ }^{[13]}$ ) can be employed to pattern the ZIF-8 crystals in a regular manner. However, ZIF-8 structures fabricated using those lithography techniques are normally in colloidal forms aggregated by multiple ZIF-8 crystals ${ }^{[12,13]}$ and the critical dimension is normally in micrometer scale, which is determined intrinsically by diffraction resolution limit. 
It has been shown that, by integrating ZIF-8 nanofilms with plasmonic materials, e.g. gold or silver nanostructures, the plasmonic adsorption can be modulated and thereby realizing a photochemical sensor. ${ }^{[14]}$ A more recent work ${ }^{[15]}$ demonstrated the large optical nonlinearity of ZIF-8 material, making it a promising candidate for many emerging optical applications. In this paper, we explored the resonant optical properties of wafer-based isolated ZIF-8 crystals at nanoscale dimensions. The ZIF-8 structures were converted from ZnO films deposited using ALD, and gold nanoparticles (Au NPs) were used to confine the crystallization of ZIF-8 structures, thus in a way "patterning” the ZIF-8 structures and thereby resulting in isolated ZIF-8 nanocrystals. The smallest size of the fabricated ZIF-8 crystals can be scaled down to $100 \mathrm{~nm}$ with well-defined rhombohedral or rhombic dodecahedron morphologies. The synthesized ZIF-8 nanocrystals exhibited broadband scattering resonances in visible wavelengths, which gave prominent structural colors. Besides, an evident redshift of structural colors was observed when the sizes of single ZIF-8 nanocrystals increase. Such size-dependent resonant scattering behavior is of central importance for the applications in nanophotonics and bionanotechnology. ${ }^{[16-20]}$ By presenting a facile fabrication method of wafer-based ZIF-8 nanocrystals and revealing their fundamental light scattering properties, we believe our work can provide new possibilities for dielectric ZIF-8 nanostructures functionalized with favorable optical properties.

\section{Results and discussion}

Figure 1 shows a schematic illustration of the process flow, in which three steps are included to fabricate ZIF-8 nanocrystals, i.e. growth of ZnO film via ALD, dispersion of Au NPs, and subsequent solvothermal conversion. The representative scanning electron microscopy (SEM) images of the samples are shown in Figure 2. The thickness of the initial $\mathrm{ZnO}$ film is determined to be $157 \mathrm{~nm}$ after 1000 cycles of deposition, and the root mean squared (RMS) roughness was $\sim 6 \mathrm{~nm}$ (Figure 2a, Figure S1a, 2a, Supporting Information). Without the addition of Au NPs, the surface of ZnO was fully transformed into a ZIF-8 colloidal film, 
with a normal size distribution and an average grain size of $~ 168 \mathrm{~nm}$ as shown in Figure 3a. Here, the size of ZIF-8 nanocrystals is defined as the side length in our discussions and following simulations. The surface and cross section morphology of ZIF-8 colloidal film was directly mapped with atomic force microscopy (AFM, Figure S1b, Supporting Information) and SEM (Figure S2b, Supporting Information). The transformation from the pristine ZnO to ZIF-8 colloidal film was also confirmed by both surface sensitive X-ray photoelectron spectroscopy (XPS) element analysis (Figure S3, Supporting Information) and energy dispersive X-ray spectroscopy (EDX) measurements (Figure S2c, Supporting Information). The XPS results suggest the existence of carbonates and water/hydroxyl groups on the film surface, and the EDX spectra verify the existence of carbon and nitrogen elements in the converted sample. The elemental analysis is in good agreement with previous studies. ${ }^{[21]}$ When Au NPs with different concentrations (20, 40, and 60 vol. \%) were pre-dispersed on ZnO film, the sizes of ZIF-8 nanocrystals exhibited a bipolar distribution, which consists of larger crystals with average size of $\sim 600 \mathrm{~nm}$ and smaller ones with mean size of $\sim 200 \mathrm{~nm}$ (Figure 3a). Moreover, the proportion of smaller crystals increases with the increasing of Au NPs concentration (Figure 3b). The similar results can also be found in different ZIF-8 samples that obtained by changing the thickness of initial ZnO film or substrates (Figure S4, Supporting Information). The number density of ZIF-8 nanocrystals decreases with a higher concentration of Au NPs as shown in Figure 3c, and this decreasing spatial density is verified by EDX analysis, which suggested a decreasing proportion of nitrogen and carbon elements when Au NPs with higher concentration was used (Figure 3d). The confined growth of ZIF-8 nanocrystals is further confirmed by AFM measurements (Figure S5, Supporting Information). The results show that the concentration and coverage of Au NPs on $\mathrm{ZnO}$ film are crucial for the subsequent conversion of ZIF-8 nanocrystals. For comparison, we discussed the distribution of Au NPs before solvothermal reactions (Figure S6, supporting information). 
This variation of size distribution of ZIF-8 nanocrystals is related to surface nucleation and spreading growth process. ${ }^{[22]}$ The interaction between $\mathrm{ZnO}$ film and solvents facilitates the deprotonation of MeIm ligands and thus reduces the $\mathrm{pH}$ value in solution. ${ }^{[23]}$ The solvated zinc ions, together with MeIm are two basic monomeric building units, which will be added sequentially in a metastable manner and crystallize into an enclosed framework structure. When the citrate stabilized Au NPs are introduced, the hydrogen bond donation (HBD) ability inside the solvent is limited, which prevents the coordination between Q3 $\mathrm{Zn}^{2+}$ and nitrogen from imidazole groups. Thus the crystallization of ZIF-8 is inhibited where Au NPs are present, and the nucleation and spreading process are confined in specific regions, where the influence of Au NPs is less pronounced. The particle size distributions show a clear defocusing trend with the increasing of Au NPs concentration (Figure 3a). This nonuniform size distribution can be explained by a focusing of size distribution in the early stage of crystal growth, followed by defocusing of size distributions caused by the further growth of larger ZIF-8 crystals in later stage. ${ }^{[24]}$ In our experiments, the crystal growth and coalescence processes are confined by Au NPs, which “define” the sizes of ZIF-8 nanocrystals. Since the Au NPs were manually dispersed on the $\mathrm{ZnO}$ film, the particle distribution is not perfectly uniform, which affects the growth of ZIF-8 nanocrystals and results in a dispersive size distribution. Therefore, by precisely controlling the size and location of Au NPs via the methods such as Langmuir-Blodgett assembly or spin coating, the dimension of ZIF-8 crystals can be further tuned.

For a high concentration of Au NPs of 60 vol. \%, isolated single ZIF-8 nanocrystals are formed and embedded in the substrate (Figure 4a), and the surface roughness can be clearly distinguished (Figure 4b). These nanocrystals exhibit a well-defined rhombohedral or rhombic dodecahedron morphology (Figure 4a-b), and a size of sub-100 nm could be achieved (Figure 4c). The crystallization of ZIF-8 crystals starts with a higher growth rate on $\{111\}$ crystalline planes as compared with $\{110\}$ and $\{100\}$ planes, thus giving a cubic shape exposed with 
$\{100\}$ facets. Then the growth rate on $\{100\}$ planes dominates on the other two planes, resulting in the transformation from the initial cubic form into two-fold symmetric rhombic dodecahedra structures with a higher thermal stability. ${ }^{[24,25]}$ Since ZnO layer deposited by ALD is polycrystalline, some clusters of ZIF-8 crystals could also be found (Figure 4d), indicating a coalescence-growth process of smaller ZIF-8 crystals. EDX elemental mapping analysis confirms the uniform distribution of both $\mathrm{N}$ and $\mathrm{C}$ elements inside the region of rhombic dodecahedron structures (Figure 4e). The appearance of Au NPs in the converted ZIF-8 colloidal film is examined. When the concentration of Au NPs is 20 vol. \%, the EDX signal from Au NPs is difficult to be detected, while for a high concentration of $60 \mathrm{vol.} \%$, ZIF-8 nanocrystal and Au NPs coexist as shown in Figure S7, Supporting Information. Besides Au NPs, we also studied the role of silver nanoparticles (Ag NPs, average diameter $10 \mathrm{~nm}$ ) on the conversion of ZnO to ZIF-8 crystal. Different from Au NPs, the initial ZnO film transformed uniformly into ZIF-8 crystals with the addition of Ag NPs (Figure S8, Supporting Information), i.e., no confined growth is observed in the case of Ag NPs, which may arise from the different affinity between the metal NPs and zinc-nitrogen coordination.

Next, we study the optical properties of the synthesized ZIF-8 nanocrystals by investigating their light scattering properties with dark field optical microscope. A fused silica wafer was used as the substrate, which is transparent in the ultraviolet and visible spectral ranges. A silicon carrier wafer $(150 \mathrm{~mm})$ was used to prevent the conformal growth of $\mathrm{ZnO}$ on backside of fused silica wafer. A thinner ZnO layer with a thickness of $60 \mathrm{~nm}$ was deposited in order to make sure that all the $\mathrm{ZnO}$ was reacted and the substrate was purely optically transparent silica. The surface was mapped by EDX analysis after solvothermal reaction, which shows ZIF-8 crystals and silica substrate (Figure S9, Supporting Information). A typical direct dark field image (Figure 5a) shows a variety of colors that are produced by the ZIF-8 crystals. Corresponding SEM image (Figure 5b) demonstrates the specific dimensions and orientations of the crystals, indicating a strong dependence of the different 
colors on the particle geometry. To verify this relation, dark field scattering of individual ZIF8 nanocrystal with different size was studied as illustrated in Figure 5c,d. One can clearly see that the scattered light is shifted from deep blue color to red color with the increasing sizes of the ZIF-8 nanocrystals.

To fully analyze the progressive color evolution with the crystal size, we performed finite-difference time-domain (FDTD) simulations to calculate the optical response of individual ZIF-8 nanocrystals (Figure 6). A total-field scattered-field (TSFS) method was exploited to reduce computation effort and provide both near field profiles and far-field scattering spectra. A refractive index of 1.59 was adopted for ZIF-8 nanocrystals in our simulation. ${ }^{[15,26]}$ Scattering response of rhombic dodecahedral and cubic nanocrystals are studied with different side lengths ranging from 300 to $800 \mathrm{~nm}$. We would like to note that these two shapes are intended to be chosen because the fabricated ZIF-8 crystals actually possess transitional morphologies between them. Given the high rotational symmetry of these two shapes, only specific orientations are considered for simplicity, as shown in Figure 6a. In Figure 6b,e, scattering efficiency $\mathrm{Q}_{\text {scat }}$ is defined as the ratio of the scattering cross section to the geometric cross section. When $\mathrm{Q}_{\text {scat }}>1$, it indicates an enhanced scattering process induced by an efficient light-matter interaction. This enhanced scattering phenomenon can be found in most spectral regions of ZIF-8 nanocrystals with sizes larger than $300 \mathrm{~nm}$, covering the entire visible range. Substantial red shifts can also be readily seen in these scattering spectra with increasing crystal size, which is in good accordance with the above experimental observations. Given the practical constrains of our facilities to directly measure the dark-field spectra of individual ZIF-8 nanocrystals, we in turn performed proof-of-principle experiment to measure the collective response of a certain range of ZIF-8 crystals with relatively uniform size. Reflection and transmission spectra were measured by using ellipsometry and linear spectroscopy respectively, as shown in Figure S10. Due to the spatial resolution of the incident beam size, all these spectra reflect the collective response of a cluster of ZIF-8 
crystals. The variations on particle size, orientations and morphologies of the crystals would largely broaden the spectra, thereby leading to only one pronounced peak that can be readily seen. Nevertheless, all measured spectra show clear optical resonances in the UV-visible range and substantial size dependence, confirming the tunability of structural colors produced by the ZIF-8 crystals.

It is also worth mentioning that, whereas scattering and associated colors are conventional optical phenomenon for many nanoparticles, they are also one of the most fundamental and significant physical processes which can provide an effective measure to investigate the fundamental optical properties of nanomaterials. Scattering behaviors of nanoparticles have thus simulated the development of a diverse set of applications ranging from biosensing and thermos-therapy to optical antennas and information storage ${ }^{\text {[16-20, 27-30] }}$. During the past decades, the physics of light scattering by subwavelength nanoparticles were commonly associated with Rayleigh scattering or surface-plasmon interactions supported by metallic materials such as gold or silver. For non-metallic nanoparticles, it is only until very recently that people started to investigate their scattering properties and relevant optical resonances $^{[28]}$. A multitude of intriguing scattering properties of various dielectric nanoparticles have been unveiled, opening up unique possibilities for many novel applications such as color filtering, color printing and quantum information technology ${ }^{[29,30]}$. In this context, Mie theory ${ }^{[27]}$ provides a fundamental physical framework to understand the sizedependent scattering response of the ZIF-8 nanocrystals. The broadband scattering feature of the ZIF-8 nanocrystals is due to the low permittivity contrast between the crystal and the surrounding environment, which leads to a slightly dispersive polarization current inside the particles and further results in the Mie resonances and associated scattering colors. The noticeable scattering peaks can be attributed to the optically induced electric and magnetic dipolar or multipolar resonances. Figure 6c shows the near-field electric and magnetic distributions at first two peak wavelengths for rhombic dodecahedral ZIF-8 nanocrystals with 
$\mathrm{d}=500 \mathrm{~nm}$. At the first scattering resonance $\left(\lambda_{1}=820 \mathrm{~nm}\right)$, the electric field shows a clear electric dipolar behavior with two hot spots at both ends of the crystal along with the incident polarization in the $\mathrm{x}$ direction while the magnetic profile demonstrates an evident magnetic dipolar response with a strong concentration of the magnetic field inside the particle. Such a confined magnetic dipolar contribution also contributes to the shoulder ( $1020 \mathrm{~nm})$ appeared on the long-wavelength side of the peak, re-verifying the broadband optical response of the ZIF-8 nanocrystals. The coexistence of the electric and magnetic dipolar resonances has recently given rise to a variety of interesting applications such as the directional scattering, enhanced Raman spectroscopy and Huygens metasurfaces. ${ }^{[31-33]}$ Differently, at the second scattering peak $\left(\lambda_{2}=680 \mathrm{~nm}\right)$, higher-order electric and magnetic multipolar responses can be seen with more complicated field distributions. The electric standing wave and two separate magnetic maxima imply the excitation of a hybrid Fabry-Perot-Mie resonance. ${ }^{[34,35]}$

Similar electric and magnetic-type Mie resonances are also possessed by the cubic nanocrystals (Figure 6d-f), with relatively smaller magnitudes and blue-shifted spectra. We also plot two-dimensional maps of the scattering efficiency as a function of the incident wavelength and the crystal size for both morphological nanocrystals (see Figure S11, Supporting Information). The scattering peak wavelengths exhibit a linear red-shift tendency with respect to the increasing crystal size. Given the wide range of the crystal size that we can obtain, we may expect that the intriguing scattering resonances of the ZIF-8 crystals can be further extended from the visible spectrum to the infrared region. The influence of the incident polarization is additionally studied. As shown in Figure S12, Supporting Information, there is only a slight difference between two orthogonal polarizations due to the highrotational symmetry of the synthesized crystals.

The presented structural color and the fascinating scattering properties provide a credible new pathway for ZIF-8 crystals to be used in a range of nanophotonic applications. For example, the broadband and efficient light-matter interactions and the induced optical 
magnetism make the ZIF-8 a promising low-index optical material that can be applied in the visible metasurfaces and planar optics. ${ }^{[36-39]}$ It is also worth re-emphasizing the significant chemical features of ZIF-8 material, including thermal stability, chemical tolerability and selective-sorption properties. We believe that the combination of such remarkable chemical properties and its unique optical characteristics can further bring entirely novel and unexplored possibilities in a diverse set of interdisciplinary applications such as photochemical catalysis, chemical sensing and spectral imaging.

\section{Conclusions}

In summary, we have demonstrated a wafer based fabrication method for isolated ZIF-8 nanocrystals, and the zinc source was provided by zinc oxide thin film deposited by ALD. To selectively convert zinc oxide thin film into isolated ZIF-8 nanocrystals, we used Au NPs as an inhibitor for the crystallization process, which could confine the density and size of ZIF-8 nanocrystals depending on the concentration of Au NPs. The light scattering properties of ZIF-8 nanocrystals in visible wavelengths and size induced redshift of resonance were observed directly by dark field optical microscope. This resonant optical response was verified by FDTD simulation, which further suggested a broadband resonance of ZIF-8 nanocrystals in both visible and infrared wavelengths depending on geometry and sizes. The fabrication method we proposed provides new possibilities not only to study ZIF-8 crystals, but also to integrate this potential material with various micro and nanofabrication techniques. The structural colors induced by Mie resonances also suggest ZIF-8 as a promising dielectric material for nanophotonics research, which can be combined with chemical or biological applications.

\section{Experimental Section}


Deposition of ZnO film: ZnO films were deposited with a thermal ALD system (Picosun R200). The substrates used were (100) silicon wafers (100 mm, single side polished, with native oxide) or fused silica wafers (100 mm, double side polished). The layer by layer ALD was realized by subsequently applying two precursors - diethylzinc $\left(\mathrm{Zn}\left(\mathrm{C}_{2} \mathrm{H}_{5}\right)_{2}\right.$, DEZ) and deionized water, with a carrier gas $\left(\mathrm{N}_{2}\right)$ flow of $150 \mathrm{sccm}$ and $200 \mathrm{sccm}$, respectively. The pulse times of both precursors were $0.1 \mathrm{~s}$, the purge time for water was $0.5 \mathrm{~s}$, while the purge time for DEZ was set to be 20 s to remove any reaction byproducts and unreacted precursors. Since a low deposition temperature can give better structural and optical qualities of $\mathrm{ZnO}$ thin film, ${ }^{[40]}$ the reaction temperature was set to be $200{ }^{\circ} \mathrm{C}$, under which the deposition rate was suggested to be $0.15 \mathrm{~nm} /$ cycle. $^{[41]}$

Conversion ZnO films into ZIF-8 crystals: Au NPs (20 $\mu \mathrm{L}$; OD1, average diameter $10 \mathrm{~nm}$, stabilized suspension in $0.1 \mathrm{mM}$ PBS, Sigma-Aldrich) with concentrations of $20 \mathrm{vol}$. $\%, 40$ vol. \% and 60 vol. \% were dispensed on the top of deposited $\mathrm{ZnO}$ layer. The samples were dried and cleaved into small pieces with size of around $1 \mathrm{~cm}$ by $1 \mathrm{~cm}$. After that deionized water (DIW) was used to rinse the sample surface for 1-2 minutes to remove possible potassium chloride residues in the PBS buffer solution. The distribution of Au NPs was analyzed with SEM and an image processing procedure as discussed in Figure S6, Supporting Information. The ZnO thin film was transformed into ZIF-8 crystals through solvothermal reaction. In a typical process, dimethylformamide (DMF, $45 \mathrm{~mL}$ ) was mixed with 2methylimidazole (0.2 g) and DIW (15 mL). ZnO thin films were then immersed in the mixed solution and placed inside a convection oven for $20 \mathrm{~h}$ at $75^{\circ} \mathrm{C}$. After the reaction, the samples were rinsed with DIW several times and dried for further characterizations.

Characterization and simulation: The sample thickness was measured by spectroscopic ellipsometry (Ellipsometer VASE, J.A.Woolam). The morphology and surface roughness were studied by SEM (Supra 60VP, Zeiss) and AFM (ICON PT, Bruker). The elemental composition was analyzed by EDX and XPS (ThermoScientific). The light scattering 
properties were studied by employing dark field optical microscope (Nikon ECLIPSE L200N), randomly polarized white light was used to illuminate the sample and the scattered light was collected normally to the substrate through a $20 \times$ dark field objective lens $(\mathrm{NA}=0.8)$. The optical response was calculated by FDTD simulations (Lumerical FDTD Solutions, version 8.11).

\section{Acknowledgements}

Financial supports from the Chinese National Natural Science Foundation (51401114) to H. Sun the Danish Research Council for Technology and Production (12-126194) to K. Mølhave are acknowledged.

Received: ((will be filled in by the editorial staff))

Revised: ((will be filled in by the editorial staff)) Published online: ((will be filled in by the editorial staff))

\section{References}

[1] L. Zhang, Z. Su, F. Jiang, L. Yang, J. Qian, Y. Zhou, W. Li and M. Hong, Nanoscale 2014, 6, 6590-6602.

[2] U. P. N. Tran, K. K. A. Le and N. T. S. Phan, ACS Catal. 2011, 1, 120-127.

[3] S. Eslava, L. Zhang, S. Esconjauregui, J. Yang, K. Vanstreels, M. R. Baklanov and E. Saiz, Chem. Mater. 2013, 25, 27-33.

[4] Q. Song, S. K. Nataraj, M. V. Roussenova, J. C. Tan, D. J. Hughes, W. Li, P. Bourgoin, M. A. Alam, A. K. Cheetham, S. A. Al-Muhtaseb and E. Sivaniah, Energy Environ. Sci. 2012, 5, 8359-8369.

[5] M. Zhu, S. R. Venna, J. B. Jasinski and M. A. Carreon, Chem. Mater. 2011, 23, 35903592.

[6] K. S. Park, Z. Ni, A. P. Côté, J. Y. Choi, R. Huang, F. J. Uribe-Romo, H. K. Chae, M.

O’Keeffe and O. M. Yaghi, PNAS 2006, 103, 10186-10191. 
[7] Y.-R. Lee, M.-S. Jang, H.-Y. Cho, H.-J. Kwon, S. Kim and W.-S. Ahn, Chem. Eng. J. 2015, 271, 276-280.

[8] N. Yanai, M. Sindoro, J. Yan and S. Granick, J. Am. Chem. Soc. 2012, 135, 34-37.

[9] S. Tanaka, K. Kida, M. Okita, Y. Ito and y. Miyake, Chem. Lett. 2012, 41, 1337-1339.

[10] K. Khaletskaya, S. Turner, M. Tu, S. Wannapaiboon, A. Schneemann, R. Meyer, A.

Ludwig, G. Van Tendeloo and R. A. Fischer, Adv. Funct. Mater. 2014, 24, 4804-4811.

[11] I. Stassen, M. Styles, G. Grenci, H. V. Gorp, W. Vanderlinden, S. D. Feyter, P. Falcaro,

D. D. Vos, P. Vereecken and R. Ameloot, Nature Mater. 2016, 15, 304-310.

[12] G. Lu, O. K. Farha, W. Zhang, F. Huo and J. T. Hupp, Adv. Mater. 2012, 24, 3970-3974.

[13] O. Dalstein, D. R. Ceratti, C. Boissière, D. Grosso, A. Cattoni and M. Faustini, Adv.

Funct. Mater. 2016, 26, 81-90.

[14] G. Lu and J. T. Hupp, J. Am. Chem. Soc. 2010, 132, 7832-7833.

[15] S. Van Cleuvenbergen, I. Stassen, E. Gobechiya, Y. Zhang, K. Markey, D. E. De Vos, C. Kirschhock, B. Champagne, T. Verbiest and M. A. van der Veen, Chem. Mater. 2016, 28, 3203-3209.

[16] B. J. Berne and R. Pecora, Dynamic light scattering: with applications to chemistry, biology, and physics, Courier Dover Publications, 2000.

[17] A. R. Parker, J. Opt. A: Pure Appl. Opt. 2000, 2, R15-R28.

[18] Y. Huang, A. R. Ferhan and D.-H. Kim, Nanoscale 2013, 5, 7772-7775.

[19] Y. Gu, L. Zhang, J. K. W. Yang, S. P. Yeo and C.-W. Qiu, Nanoscale 2015, 7, 64096419.

[20] N. Bontempi, K. E. Chong, H. W. Orton, I. Staude, D-Y. Choi, I. Alessandri, Y. S. Kivshar and D. N. Neshev, Nanoscale 2017, 9, 4972-4980.

[21] F. Tian, A. M. Cerro, A. M. Mosier, H. K. Wayment-Steele, R. S. Shine, A. Park, E. R. Webster, L. E. Johnson, M. S Johal and L. Benz, J. Phys. Chem. C 2014, 118, 14449-14456. 
[22] P. Y. Moh, P. Cubillas, M. W. Anderson and M. P. Attfield, J. Am. Chem. Soc. 2011, 133, 13304-13307.

[23] E. L. Bustamante, J. L. Fernández and J. M. Zamaro, J. Colloid Interf. Sci. 2014, 424, 37-43.

[24] J. Cravillon, R. Nayuk, S. Springer, A. Feldhoff, K. Huber and M. Wiebcke, Chem. Mater. 2011, 23, 2130-2141.

[25] C. Avci, J. Ariñez-Soriano, A. Carné-Sánchez, V. Guillerm, C. Carbonell, I. Imaz and D. Maspoch, Angew. Chem. Int. Ed. 2015, 54, 14417-14421.

[26] J. Cookney, W. Ogieglo, P. Hrabanek, I. Vankelecom, V. Fila and N. E. Benes, Chem. Commun. 2014, 50, 11698-11700.

[27] C. F. Bohren and D. R. Huffman, Absorption and scattering of light by small particles, John Wiley \& Sons, 2008.

[28] B. Luk’yanchuk, N. I. Zheludev, S. A. Maier, N. J. Halas, P. Nordlander, H. Giessen and C. T. Chong, Nature Mater. 2010, 9, 707-715.

[29] A. I. Kuznetsov, A. E. Miroshnichenko, M. L. Brongersma, Y. S. Kivshar and B. Luk’yanchuk, Science 2016, 354, aag2472.

[30] I. Staude and J. Schilling, Nature Photon, 2017, 11, 274-284.

[31] I. Staude, A. E. Miroshnichenko, M. Decker, N. T. Fofang, S. Liu, E. Gonzales, J. Dominguez, T. S. Luk, D. N. Neshev, I. Brener and Y. Kivshar, ACS Nano 2013, 7, 78247832.

[32] J. Tian, Q. Li, Y. Yang and M. Qiu, Nanoscale 2016, 8, 4047-4053.

[33] P. A. Dmitriev, D. G. Baranov, V. A. Milichko, S. V. Makarov, I. S. Mukhin, A. K. Samusev, A. E. Krasnok, P. A. Belov and Y. S. Kivshar, Nanoscale 2016, 8, 9721-9726.

[34] Y. Yang, A. E. Miroshnichenko, S. V. Kostinski, M. Odit, P. Kapitanova, M. Qiu and Y. S. Kivshar, Phys. Rev. B 2017, 95, 165426.

[35] A. Forouzmand and H. Mosallaei, Adv. Opt. Mater. 2017, 5, 1700147. 
[36] A. Zhan, S. Colburn, R. Trivedi, T. K. Fryett, C. M. Dodson and A. Majumdar, ACS Photonics 2016, 3, 209-214.

[37] S. Sun, Z. Zhou, C. Zhang, Y. Gao, Z. Duan, S. Xiao and Q. Song, ACS Nano 2017, 11, 4445-4452.

[38] F. Ding, A. Pors, S. I. Bozhevolnyi, Rep. Prog. Phys. 2017, DOI: 10.1088/1361-

6633/aa8732

[39] B. S. Luk’yanchuk, R. Paniagua-Domínguez, I. Minin, O. Minin and Z. Wang, Opt. Mater. Express 2017, 7, 1820-1847.

[40] E. Guziewicz, I. A. Kowalik, M. Godlewski, K. Kopalko, V. Osinniy, A. Wójcik, S. Yatsunenko, E. Łusakowska, W. Paszkowicz and M. Guziewicz, J. Appl. Phys. 2008, 103, 033515.

[41] E. Shkondin, O. Takayama, M. E. A. Panah, P. Liu, P. V. Larsen, M. D. Mar, F. Jensen and A. V. Lavrinenko, Opt. Mater. Express 2017, 7, 1606-1627. 


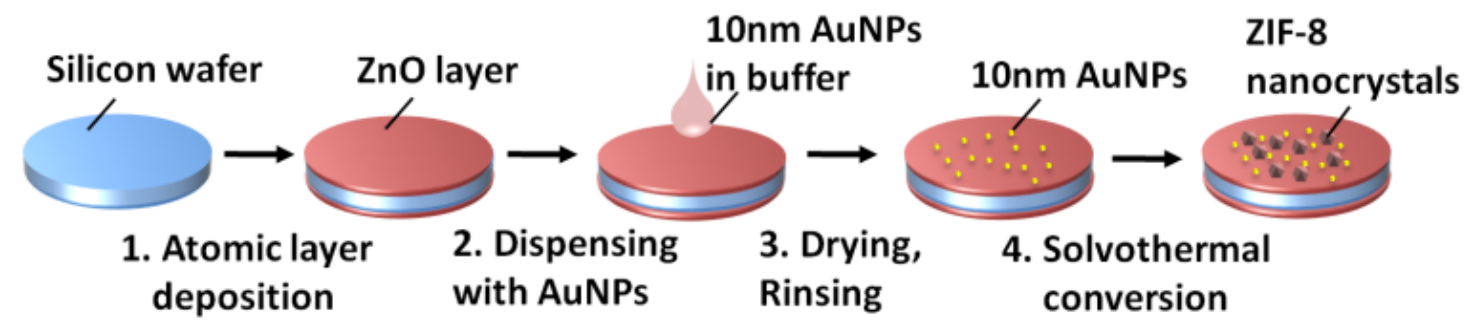

Figure 1. Schematic illustration of converting ZIF-8 nanocrystals from ALD grown ZnO layer, with steps of ALD, Au NPs dispensing and solvothermal synthesis. 


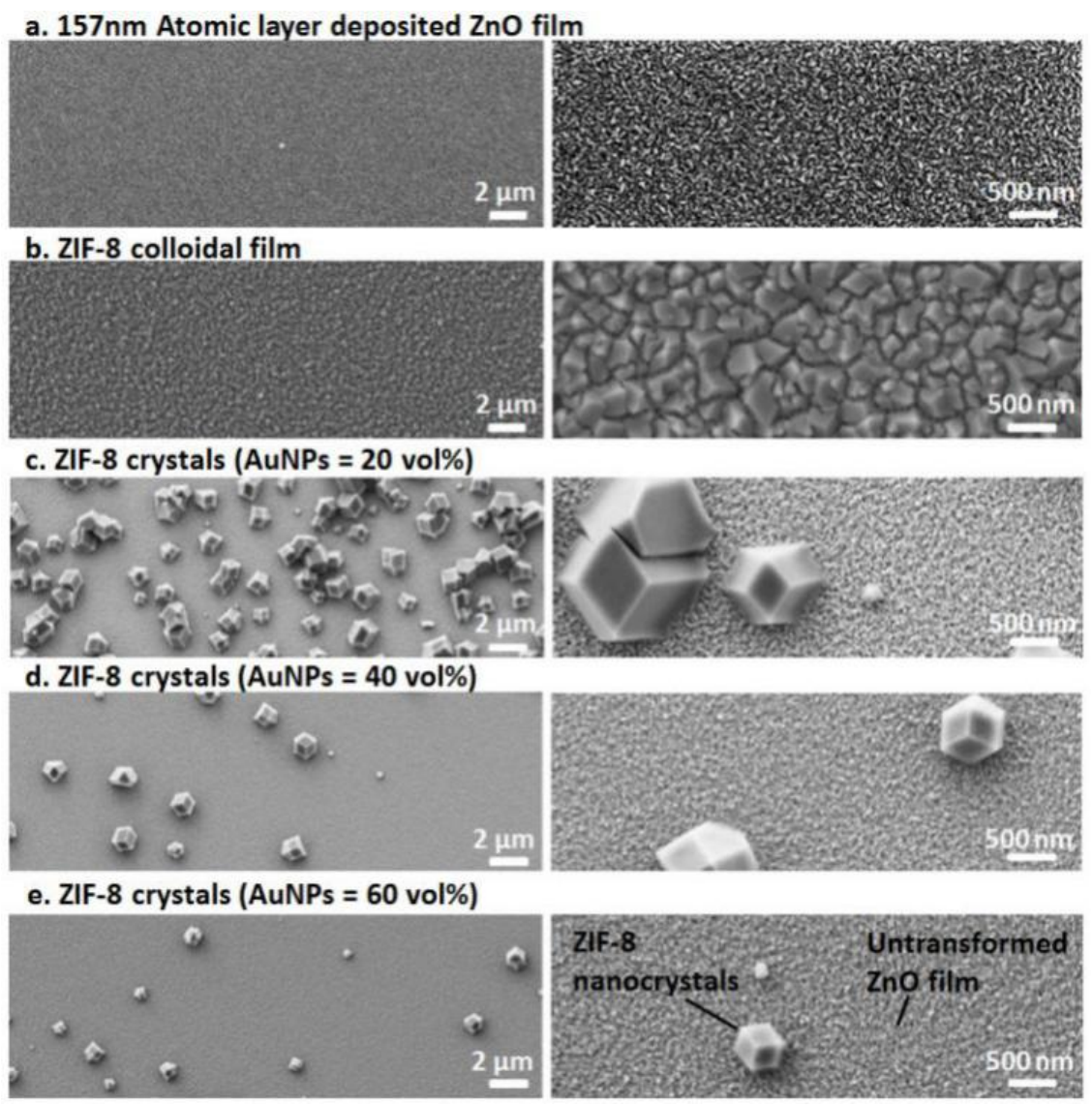

Figure 2. Low- (left column) and high- (right column) magnification SEM images of the samples. (a) $157 \mathrm{~nm} \mathrm{ZnO}$ layer deposited by ALD on silicon substrate; (b) fully transformed ZIF-8 nanocrystals from ZnO film without the addition of Au NPs; partially transformed ZIF8 crystals from ZnO film with different Au NPs concentrations: (c) 20 vol. \%, (d) 40 vol. \%, and (e) 60 vol. \%. 

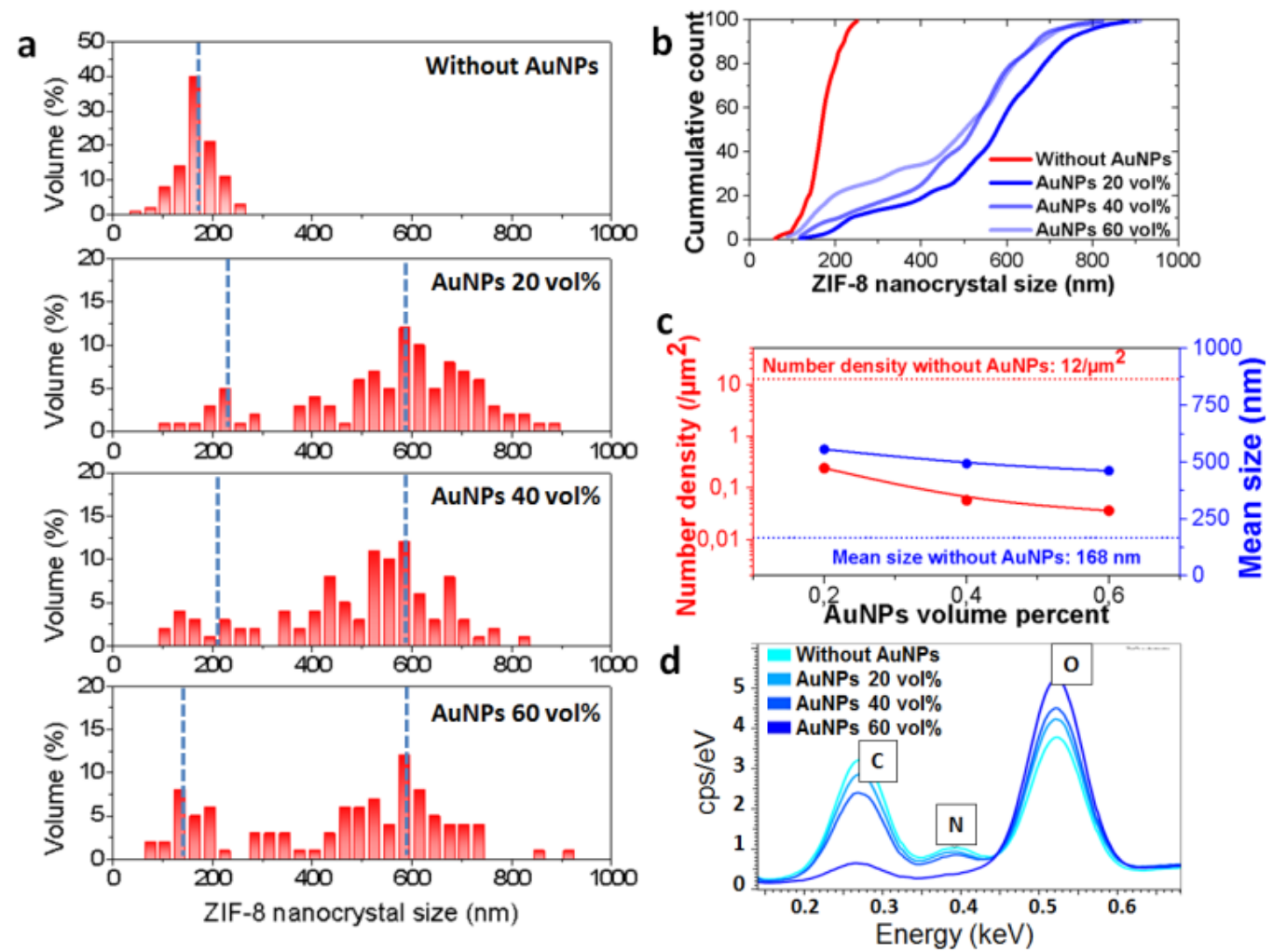

Figure 3. (a) Particle size distributions for ZIF-8 nanocrystals with different concentrations of Au NPs; (b) Cumulative plot for different concentrations of Au NPs, which shows a size reduction when the concentration of Au NPs increases; (c) Spatial densities and average sizes of ZIF-8 nanocrystals; (d) EDX element analysis of $\mathrm{C}, \mathrm{N}$ and $\mathrm{O}$, which shows a decreasing density of ZIF-8 nanocrystals when increasing the Au NPs concentrations. 

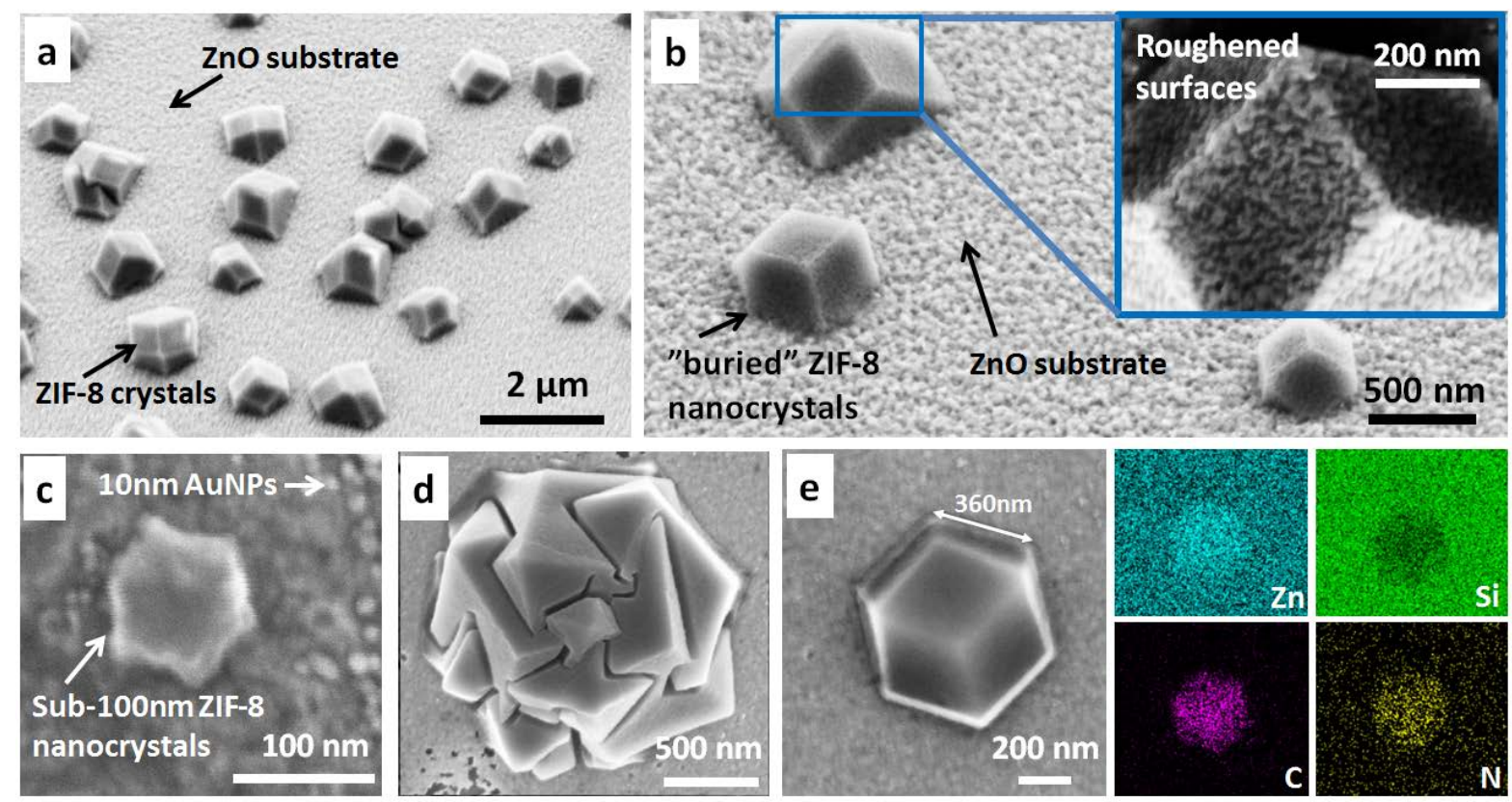

Figure 4. Surface morphology of isolated ZIF-8 nanocrystals with (a) $45^{\circ}$ tilted view of ZIF-8 crystals on the surface of $\mathrm{ZnO}$; (b) A closer view of ZIF-8 nanocrystals shows a roughened surface; (c) An isolated sub-100 nm ZIF-8 nanocrystal and $10 \mathrm{~nm}$ Au NPs; (d) Coalescence of multiple ZIF-8 crystals; (e) Asymmetric rhombic dodecahedron caused by truncated growth on [-110] direction. The EDX element mapping of a typical ZIF-8 single nanocrystal in (e), which shows the distribution of $\mathrm{Zn}, \mathrm{Si}, \mathrm{C}$ and $\mathrm{N}$ elements. The concentration of Au NPs is 60 vol. \%. 

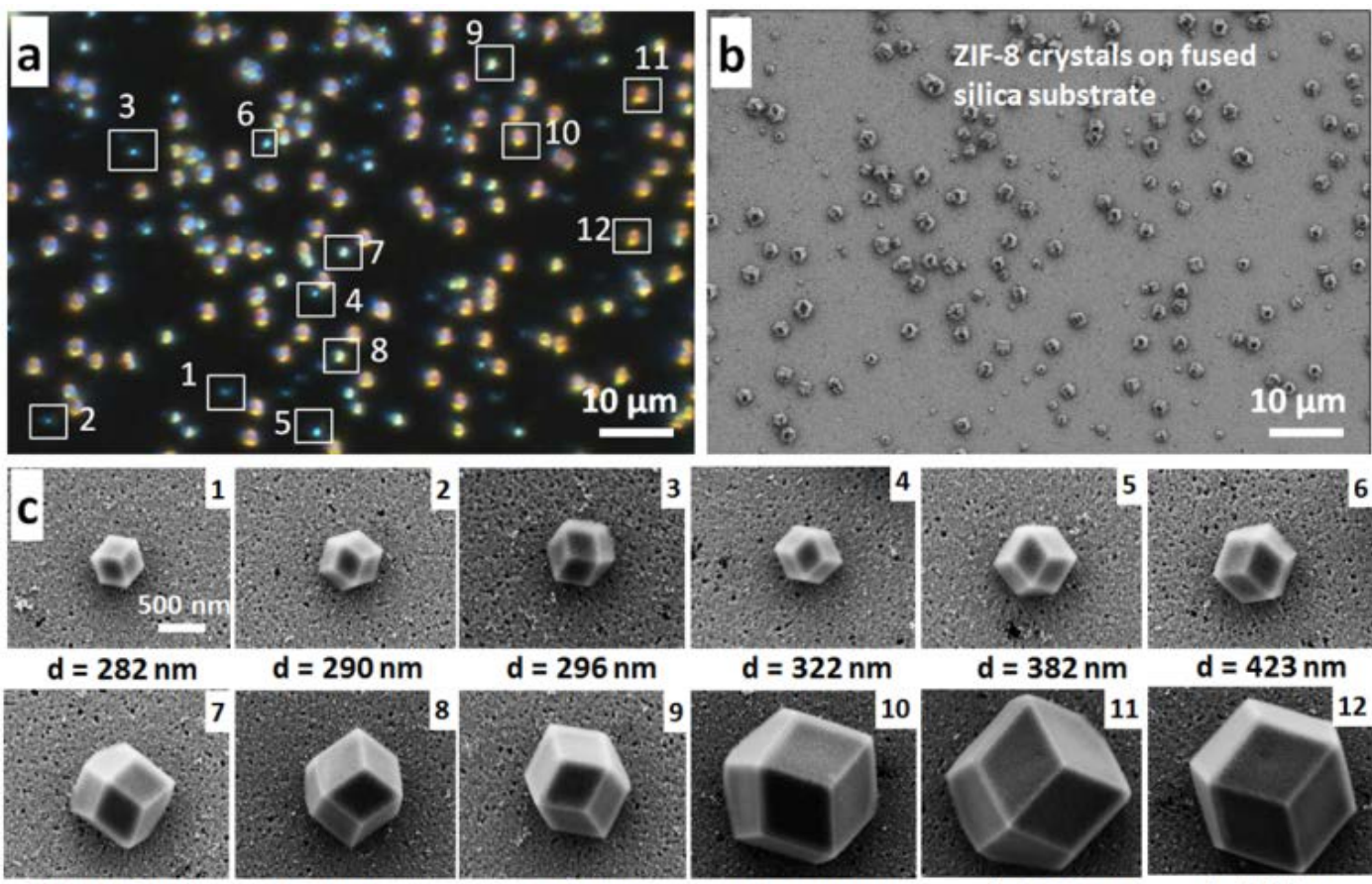

$\mathrm{d}=322 \mathrm{~nm}$

$\mathrm{d}=382 \mathrm{~nm}$

$\mathrm{d}=423 \mathrm{~nm}$

$\mathrm{d}=456 \mathrm{~nm}$

$\mathrm{d}=524 \mathrm{~nm}$
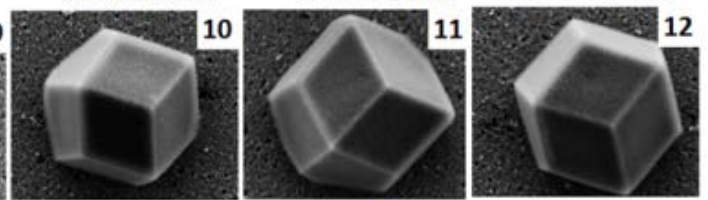

$\mathrm{d}=761 \mathrm{~nm}$

$\mathrm{d}=780 \mathrm{~nm}$

$\mathrm{d}=795 \mathrm{~nm}$

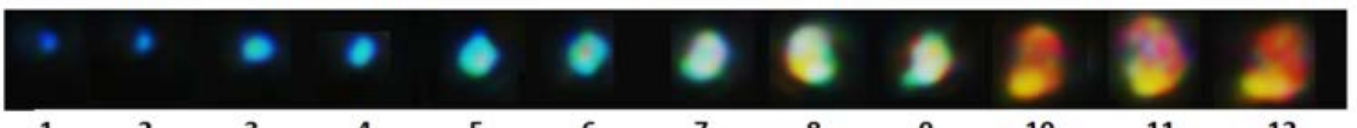

d Increasing sizes of ZIF-8 nanocrystals

Figure 5. Light scattering and structural color of ZIF-8 crystals fabricated with the addition of 60 vol. \% Au NPs. (a) Dark-field optical microscope images of ZIF-8 nanocrystals on a fused silica wafer; (b) SEM image of the corresponding area in (a); (c) SEM images of ZIF-8 nanocrystals with different size; (d) Structure colors of ZIF-8 crystals corresponding to (c). 


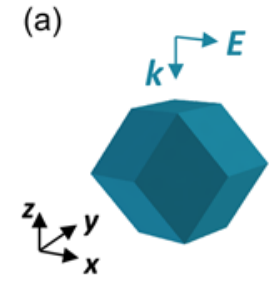

(b)

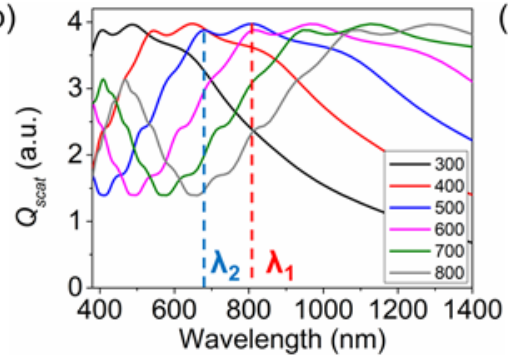

(d)

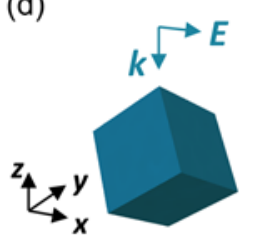

(e) (c)

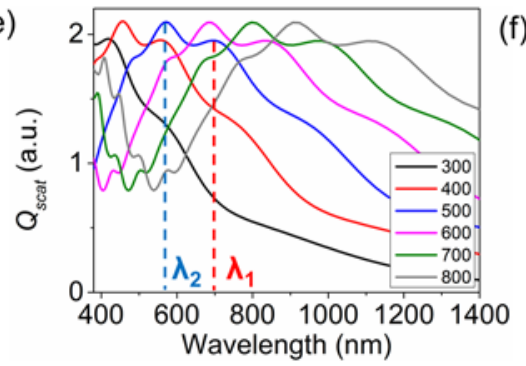

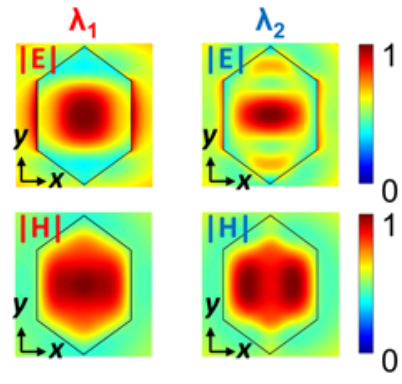
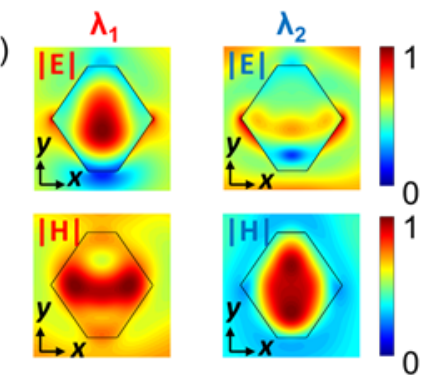

Figure 6. Calculated light scattering of rhombic dodecahedral (upper) and cubic (lower) ZIF-8 nanocrystals. (a, d) Schematic illustrations of the nanocrystals and the coordinate system. The incident polarization is in the $\mathrm{x}$ direction. (b, e) Spectral scattering efficiency of nanocrystals with different side length d ranging from $100 \mathrm{~nm}$ to $500 \mathrm{~nm}$. (c, f) Electric and magnetic near-field distributions at scattering resonances of ZIF-8 nanocrystals with a side length $\mathrm{d}=500 \mathrm{~nm}$. The $\mathrm{xy}$ profiles are taken in the middle cross section of the nanocrystals. Corresponding resonant wavelengths are labeled in panel (b) and (e) 
A novel fabrication method of ZIF-8 nanocrystals is proposed based on atomic layer deposited zinc oxide and gold nanoparticles as growth inhibitors, the fabricated ZIF-8 nanocrystals show size related structural colors, which is verified by Mie scattering theory.

Keyword: ZIF-8; gold nanoparticles; atomic layer deposition; structural colors

Bingdong Chang, Yuanqing Yang, Henri Jansen, Fei Ding, Kristian Mølhave and Hongyu Sun

\section{Confined growth of ZIF-8 nanocrystals with tunable structural colors}
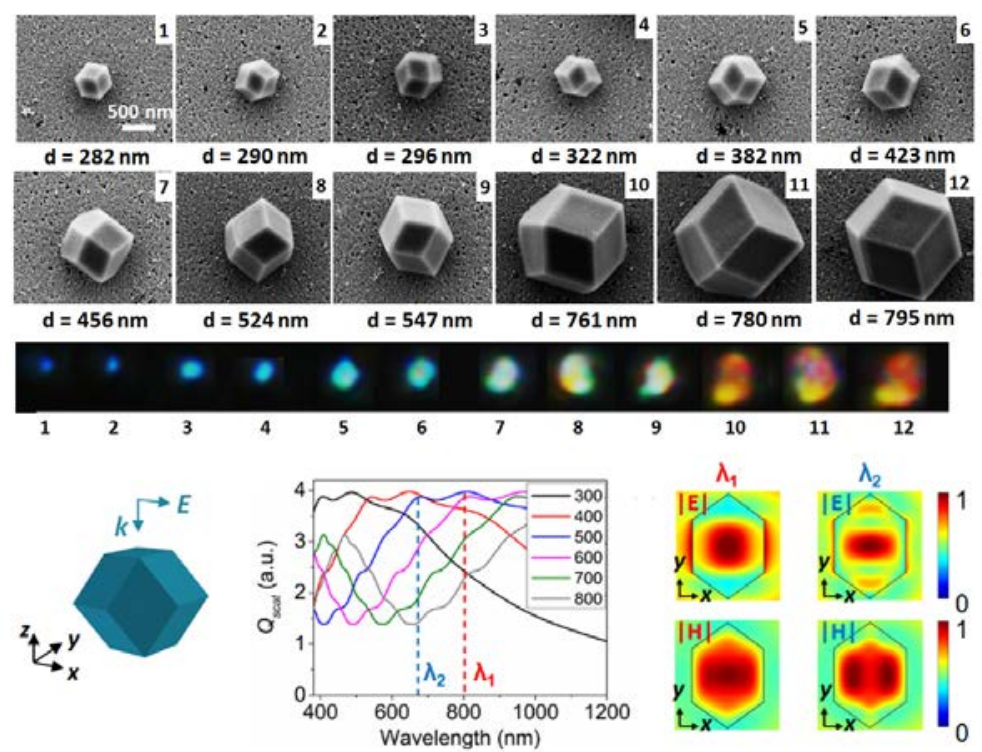


\section{Supporting Information}

\section{Confined growth of ZIF-8 nanocrystals with tunable structural colors}

Bingdong Chang, Yuanqing Yang, Henri Jansen , Fei Ding , Kristian Mølhave and Hongyu Sun 
Since it is difficult to have an accurate size control of the Au NPs by dispensing, during our experiments, the distribution of Au NPs was analyzed with SEM and image processing before solvthermal reactions, and several procedures were taken to avoid nonuniformity of $\mathrm{Au}$ NPs, e.g. the samples were cleaved into small chips of around $1 \mathrm{~cm}$ by $1 \mathrm{~cm}$, so the dispensed solution can spread over the whole surface of the chip, and nonuniformity caused by coffee ring effect could be avoided, since the edge of the dried region is outside of chip edge. The concentration of Au NPs we used was also not too high, otherwise the Au NPs will form into coilloid, and the distribution is not uniform.

Here we will give a detailed discussion of Au NPs distribution. Since Au NPs can give strong signals under secondary electron detectors on SEM, thus a good contrast between $\mathrm{Au}$ NPs and ZnO substrate can be achieved, after image processing, we can filter the signals from $\mathrm{ZnO}$ substrate (Figure S6a-b), and the fast fourier transform image suggests a random distribution of Au NPs (inset in Figure S6b). If we study the distribution of Au NPs in X-and y-directions, it also suggests a normal distribution of Au NPs in both directions (Figure S6c). By applying the image processing strategy, we can easily calculate the coverage percent of $\mathrm{Au}$ NPs on the substrate, when the concentration of Au NPs is changed. It can be seen, that the coverage percent increases linearly when the volume percent increases from $20 \%$ to $60 \%$, however, we need to be careful that when the concentration of Au NPs is too high, isolated Au NPs start to form into colloids, and the distribution is thus not uniform anymore (Figure S6d-e). Thus in our experiments, the samples were always checked first with SEM and analyzed with this image processing procedure.

\section{Reflection and transmission spectra of ZIF-8 nanocrystals}


To prove the size dependent structural colors of ZIF-8 nanocrystals, we chose the regions of ZIF-8 crystals with relatively uniform colors under dark-field optical microscope (as shown below in Figure S10 a-c), and measured the reflection and transmission spectra with ellipsometry and UV-visible spectroscopy correspondingly.

The reflection spectrum was measured by Ellipsometry (Ellipsometer VASE) as in figure S10d,e, with incident angle of 50 degrees. From the spectrum, we can see the "redshift” of structural colors of ZIF-8 crystals with respect to the crystal size. Given the spatial resolution of the incident spot size (diameter $\sim 20 \mu \mathrm{m}$ ), it is difficult to probe the scattering behavior of individual ZIF-8 crystals. The spectra actually reflect the collective optical response of a cluster of crystals with varied crystal sizes, orientations and morphologies. Therefore, only one pronounced peak can be readily seen due to the broadening effect. The transmission spectrum was obtained with UV-visible spectroscopy (UV-VIS Spectrophotometer. UV-1800, Shimadzu), with a spot size of around $1 \mathrm{~mm}$. We can also see the clear size dependence of the transmission peaks. All these measured spectra show clear optical resonances exhibited by the ZIF-8 crystals and associated size dependence, which is in reasonable agreement with simulation results. 

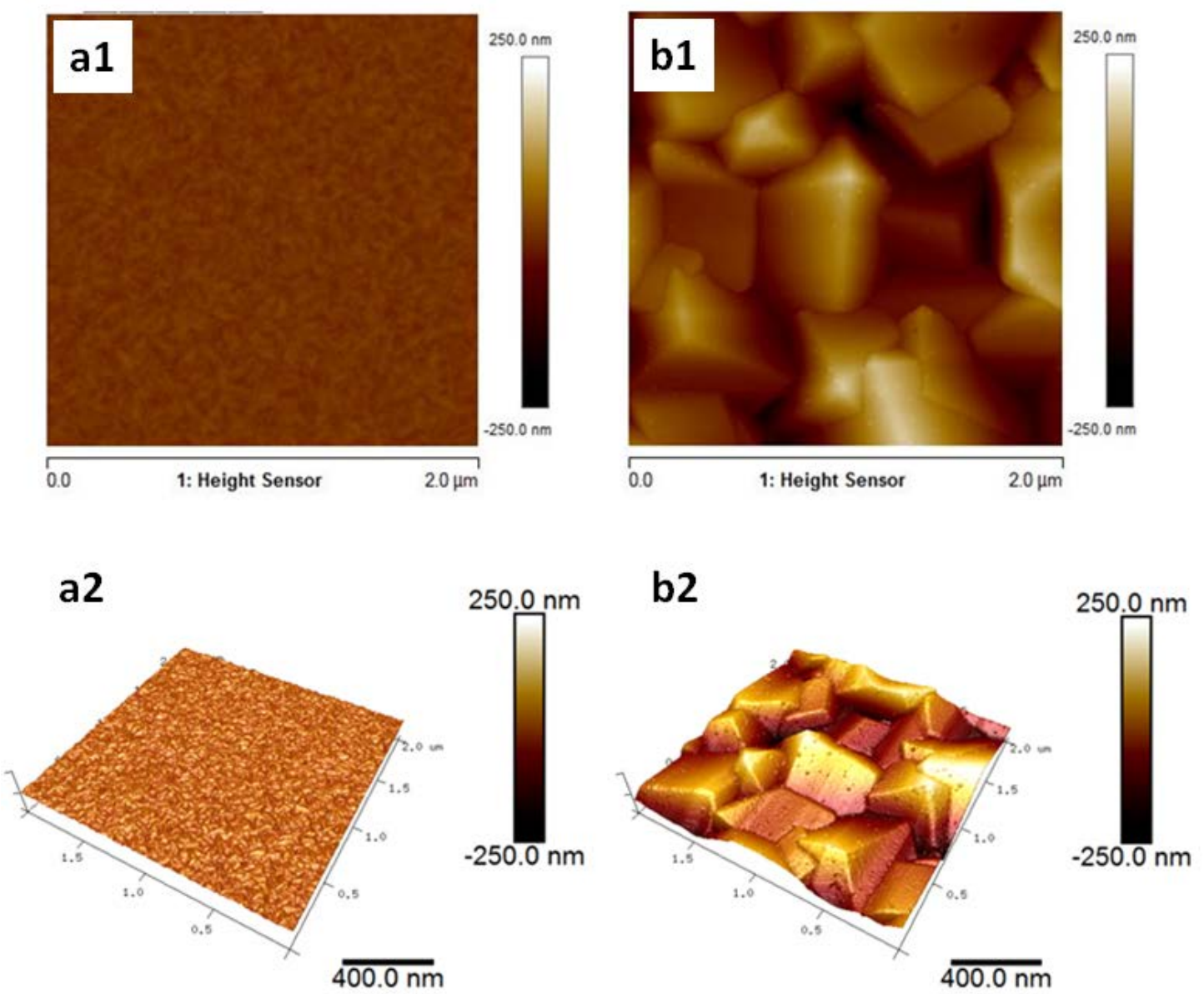

Figure S1. (a) AFM measurements of ALD deposited ZnO film in (a1) 2-dimensional view and (a2) 3-dimensional view; (b) AFM measurements of ZIF-8 colloidal film transformed from ALD deposited $\mathrm{ZnO}$ thin film in (b1) 2-dimensional view and (b2) 3-dimensional view. The measurements were carried out on AFM ICON PT (Bruker) with PeakForce Tapping ${ }^{\circledR}$ mode. 

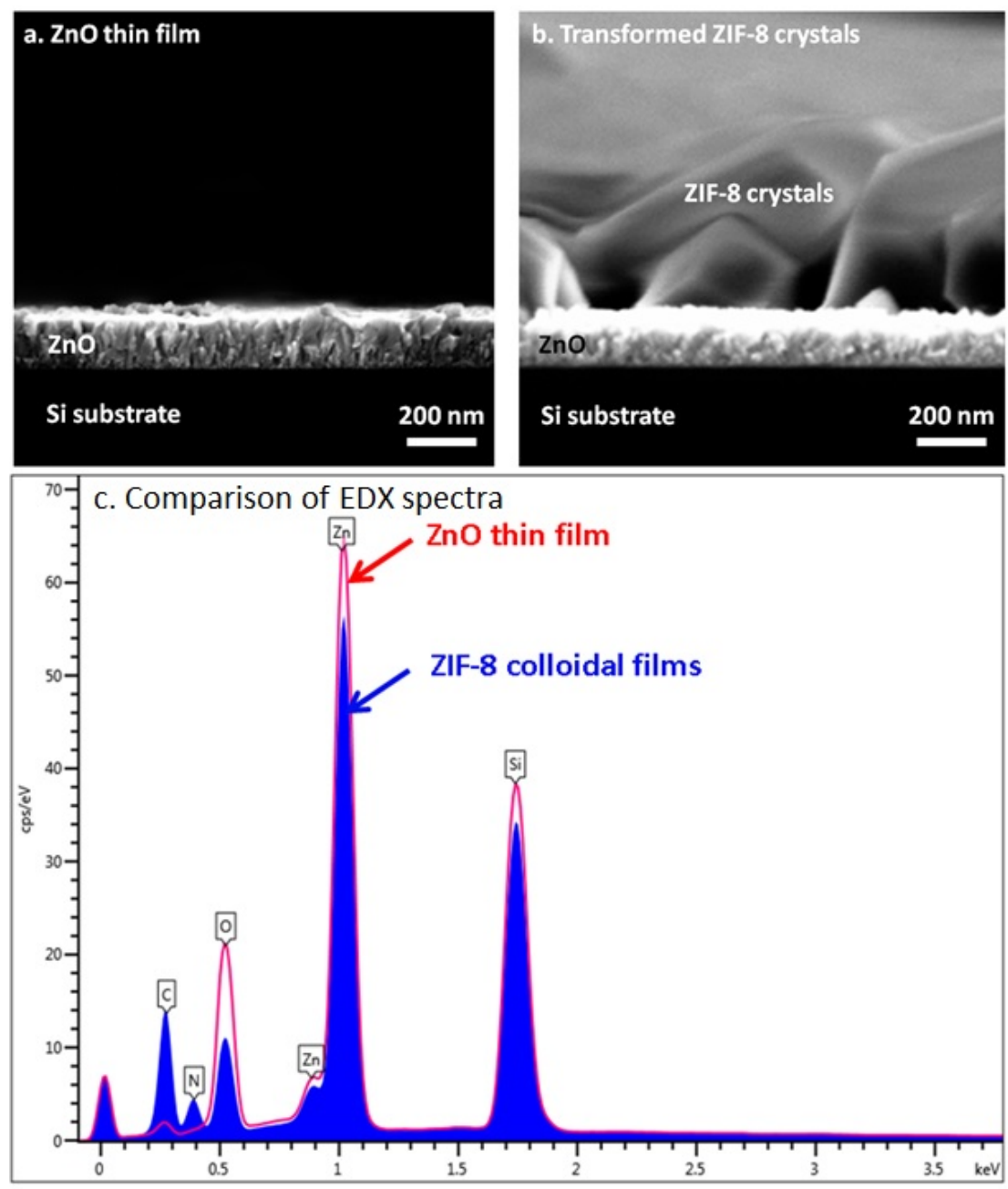

Figure S2. (a, b) Cross sectional SEM images and (c) EDX spectra of a thin ZnO layer and transformed ZIF-8 colloidal film on Si substrate. The silicon peaks in the EDX spectra come from the Si substrate. 

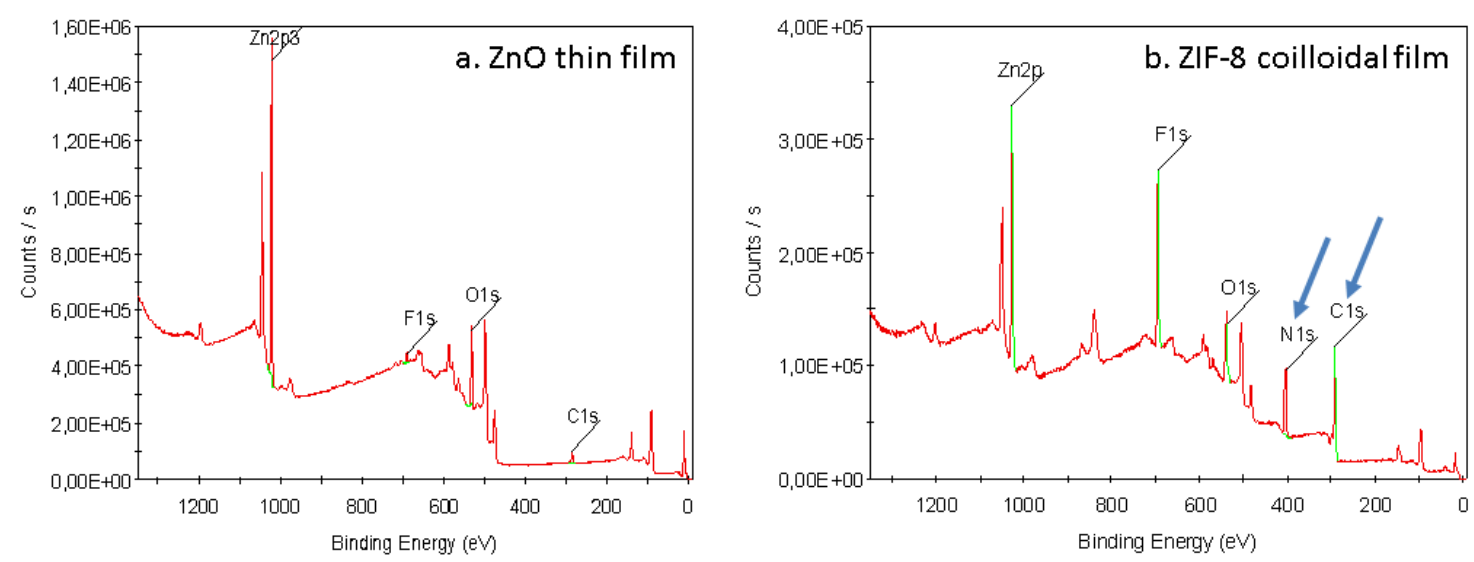

Figure S3. XPS spectra of (a) ALD deposited ZnO film and (b) transformed ZIF-8 colloidal film.

From the spectra, it can be seen the existence of nitrogen and carbon after solvothermal transformation, which is not present on the initial $\mathrm{ZnO}$ thin film. Besides, the peak positions of $\mathrm{Zn}$, carbon and oxygen are all shifted due to a change of chemical bond connections. The increasing fluorine peak is due to the containers for samples, which is well known to give fluorine contained contaminants. 
a

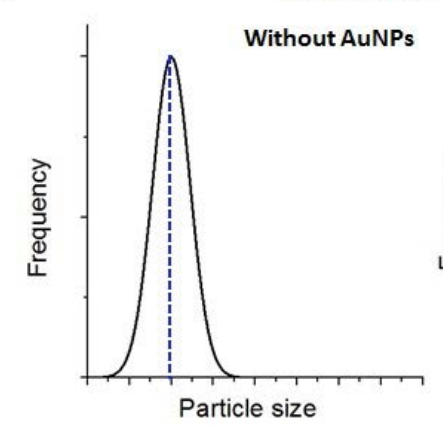

Schematic illustration of distributions of ZIF-8 crystals

b
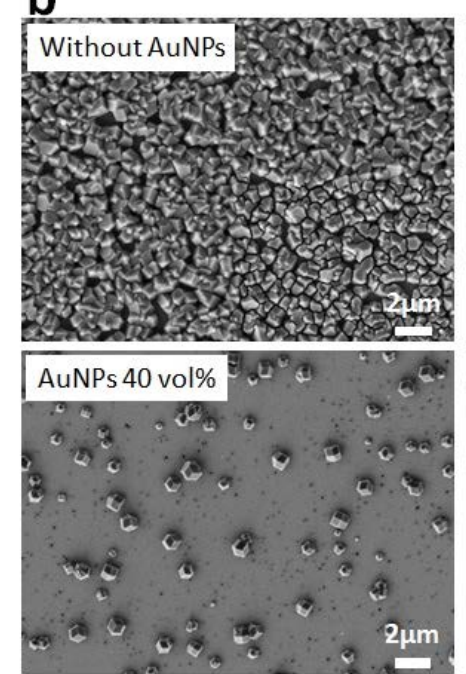

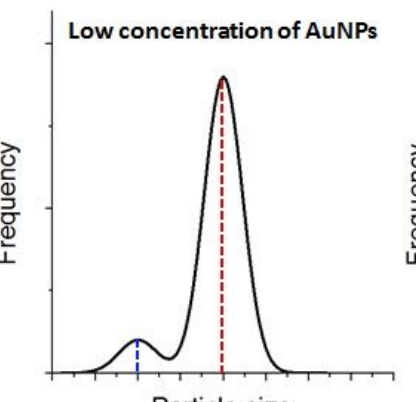

Particle size

Increased concentration of AuNPs

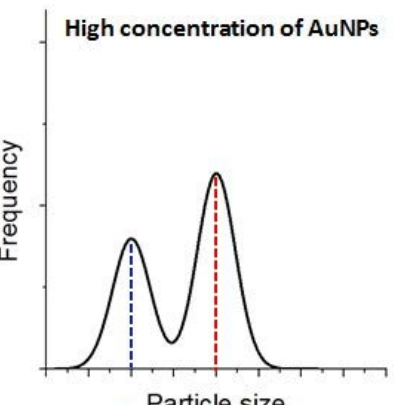

Particle size

\section{C}
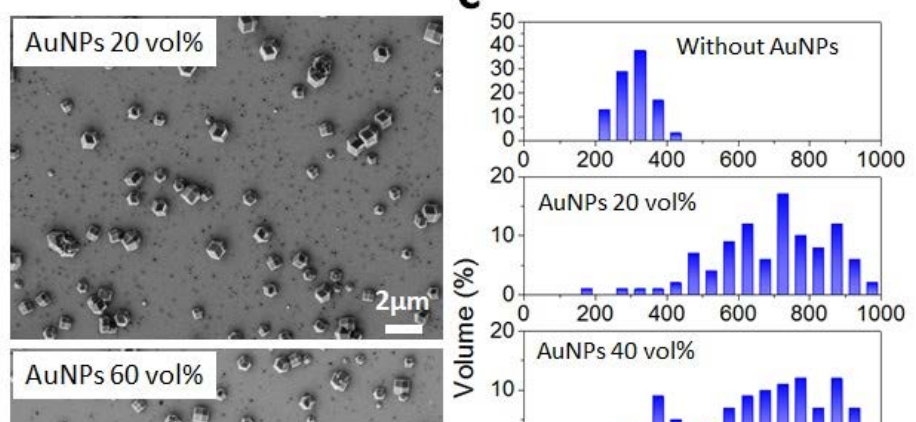

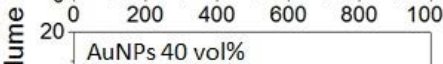

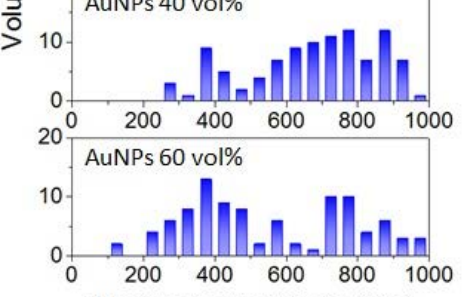

ZIF-8 nanocrystal size $(\mathrm{nm})$

Figure S4. (a) Schematic illustration of the effect of Au NPs concentration on ZIF-8 crystal size distribution; (b) SEM images of transformed ZIF-8 nanocrystals on silicon oxide substrate; (c) Particle size distribution of ZIF-8 crystals with the addition of different Au NPs concentrations. 


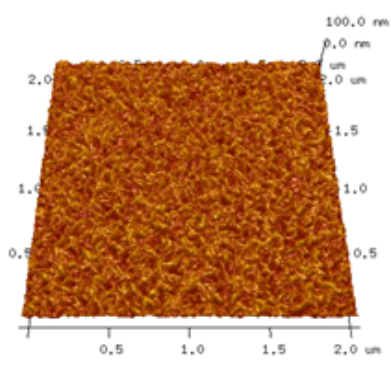

Height Sensor $4 \overline{00.0 \mathrm{~nm}}$

a. ALD grown ZnO surface

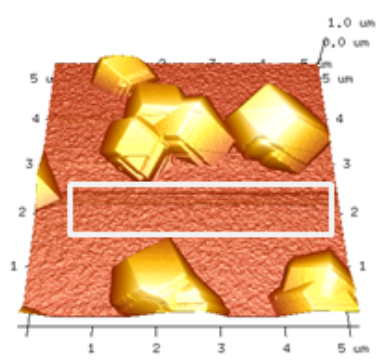

Height Sensor

$\overline{1.0 \mu \mathrm{m}}$

c. Transformed ZIF-8 crystals

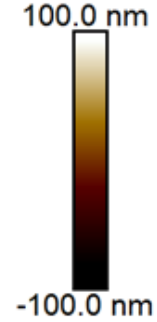

Height Sensor

$4 \overline{00.0 \mathrm{~nm}}$

b. Dispensed AuNPs on ZnO surface
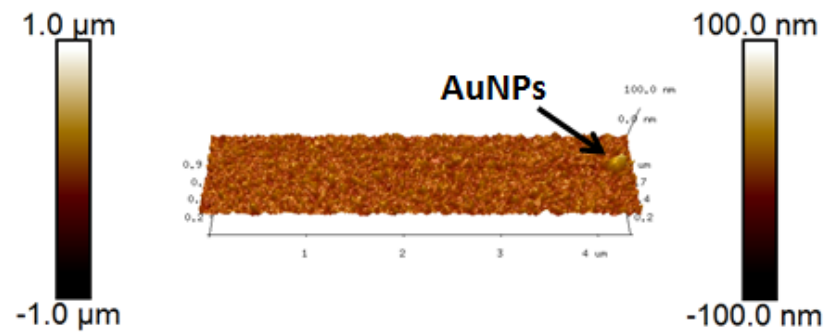

Height Sensor

$8 \overline{70.0 \mathrm{~nm}}$

d. Zoomed in from region in (c)

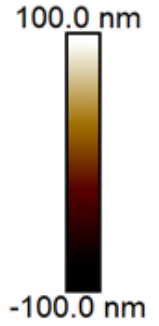

$-100.0 \mathrm{~nm}$

Figure S5. AFM measurements of ALD deposited ZnO film, and crystallization of ZIF-8 nanocrystals when the 60 vol. \% Au NPs are present. (a) ZnO surface; (b) ZnO surface with dispensed Au NPs; (c) After solvthermal reactions, ZIF-8 nanocrystals were grown on substrate, while other regions remain $\mathrm{ZnO}$; (d) A zoom in of the region from (c), suggesting the existence of Au NPs. 


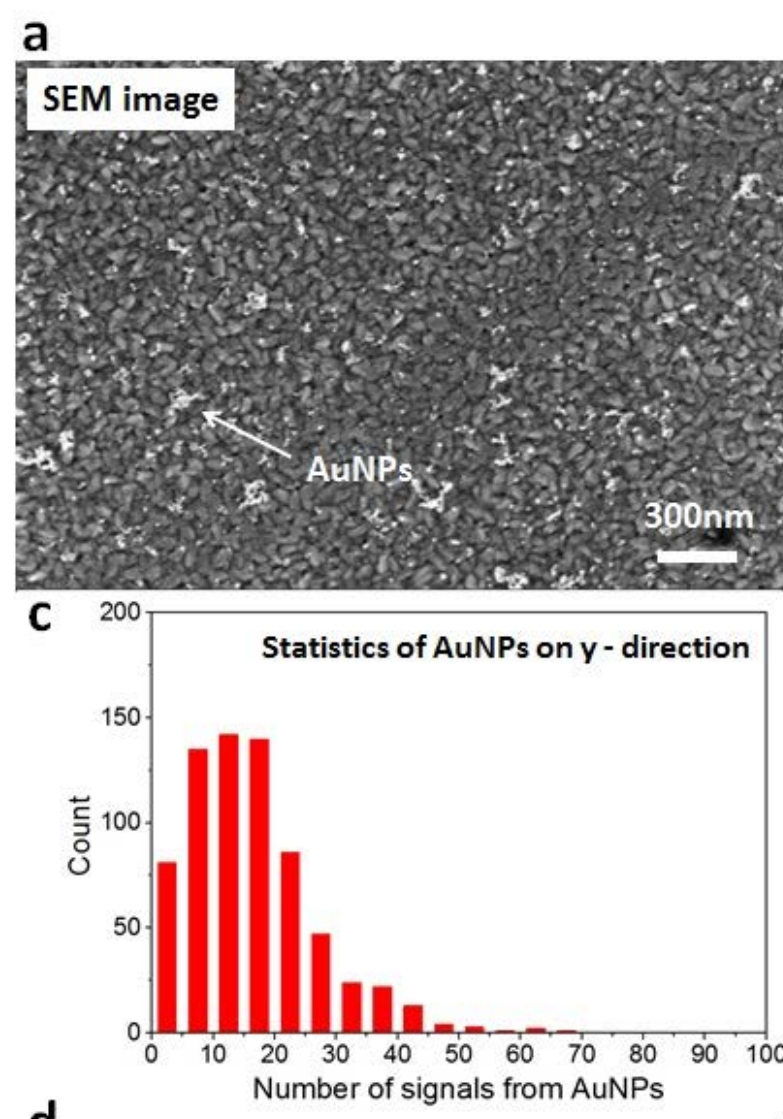

b

d
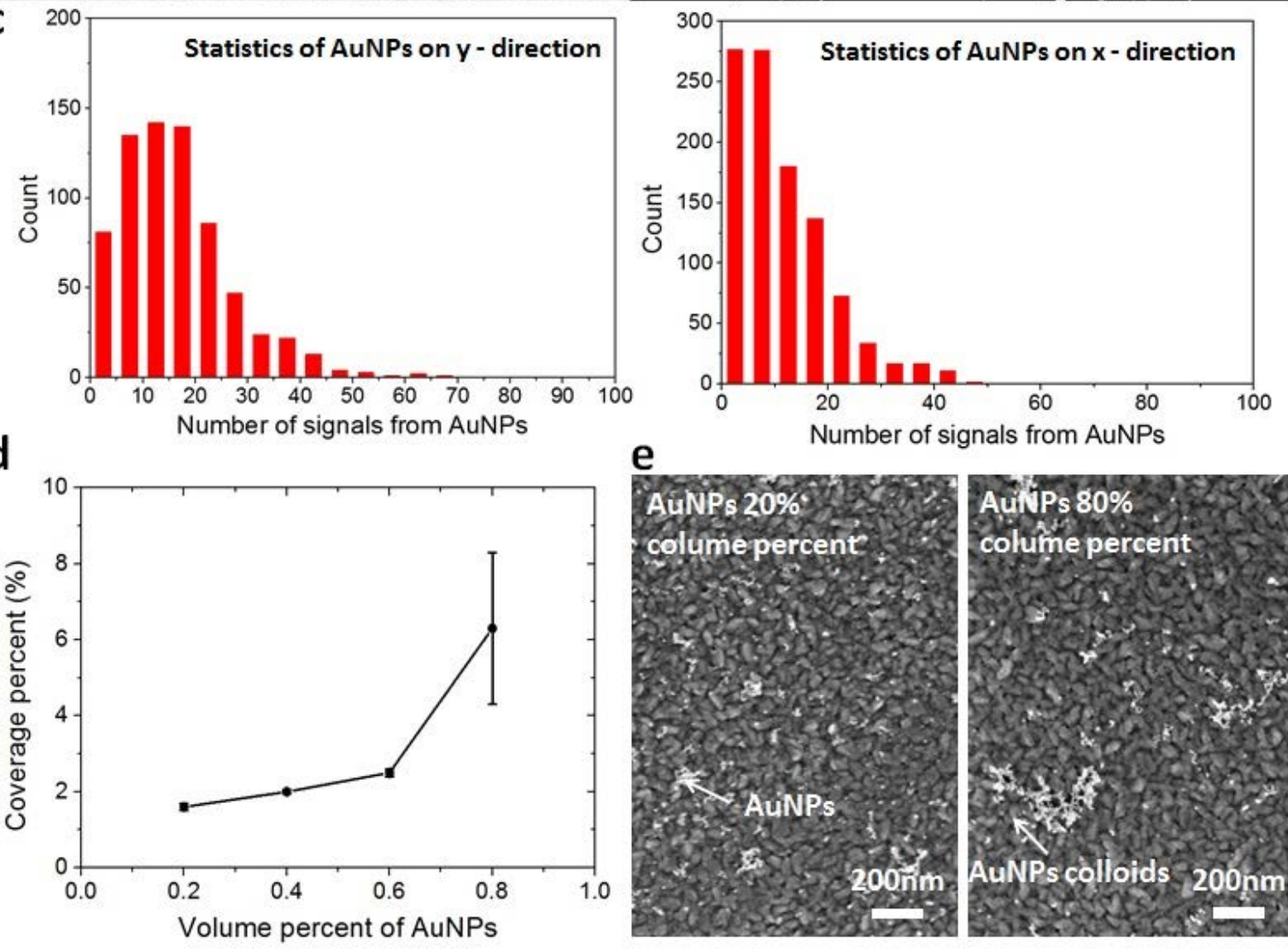

e
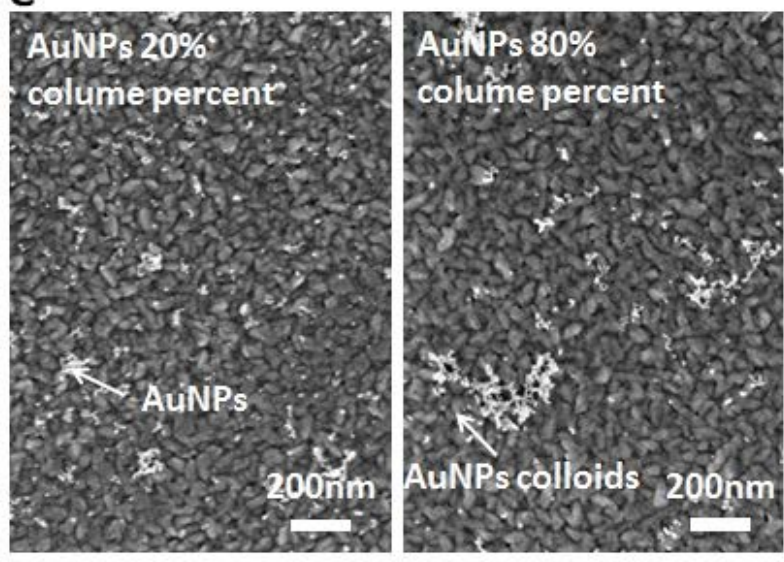

Figure S6. Size distribution of dispensed Au NPs on ZnO film before solvothermal conversion: (a) SEM image of ZnO surface with Au NPs; (b) After image processing, signals from ZnO surface are filtered, the inset is the fast Fourier transform (FFT) of Au NPs; (c) Distribution of Au NPs on x- and y-direction of the SEM image; (d) Coverage percent of Au NPs when the volume percent is increased during dispensing; (e) SEM images of ZnO surface with low (20 vol. \%) and high concentration (80 vol. \%) of Au NPs. 
Si $K \alpha 1$
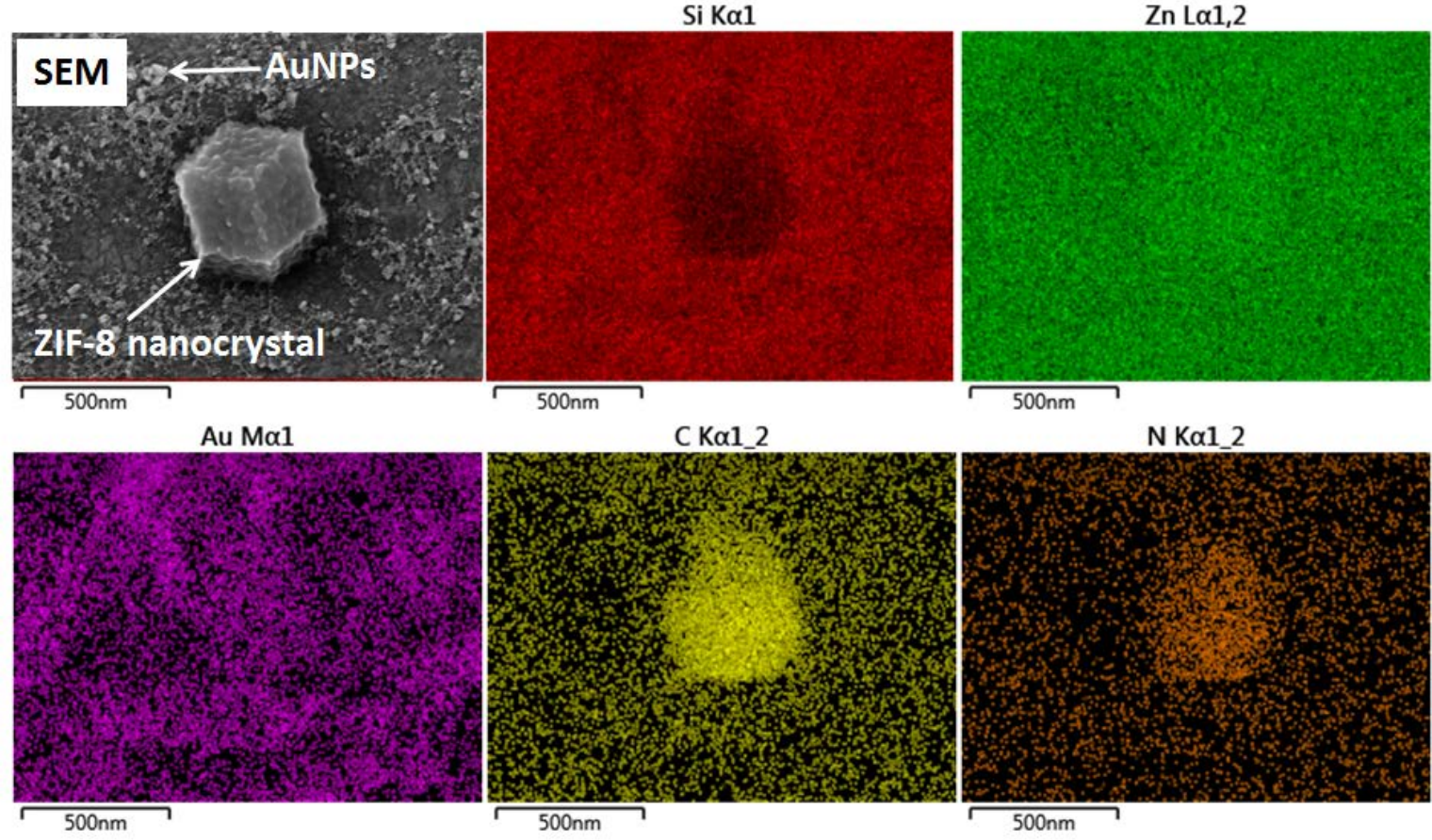

C Ka1_2

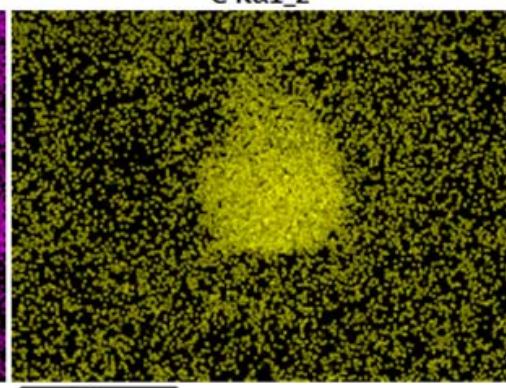

$500 \mathrm{~nm}$
N K $\alpha 1 \_2$

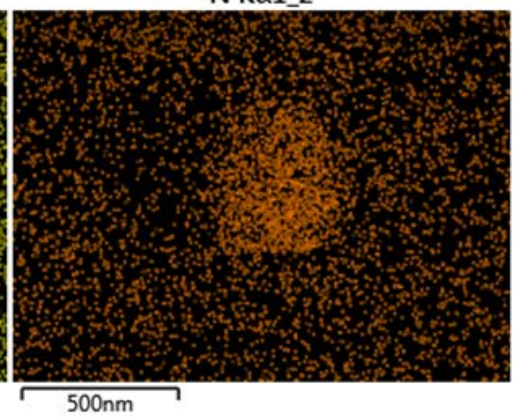

Figure S7. EDX elemental mapping of a typical ZIF-8 nanocrystal with high concentration (60 vol. \%) of Au NPs. 

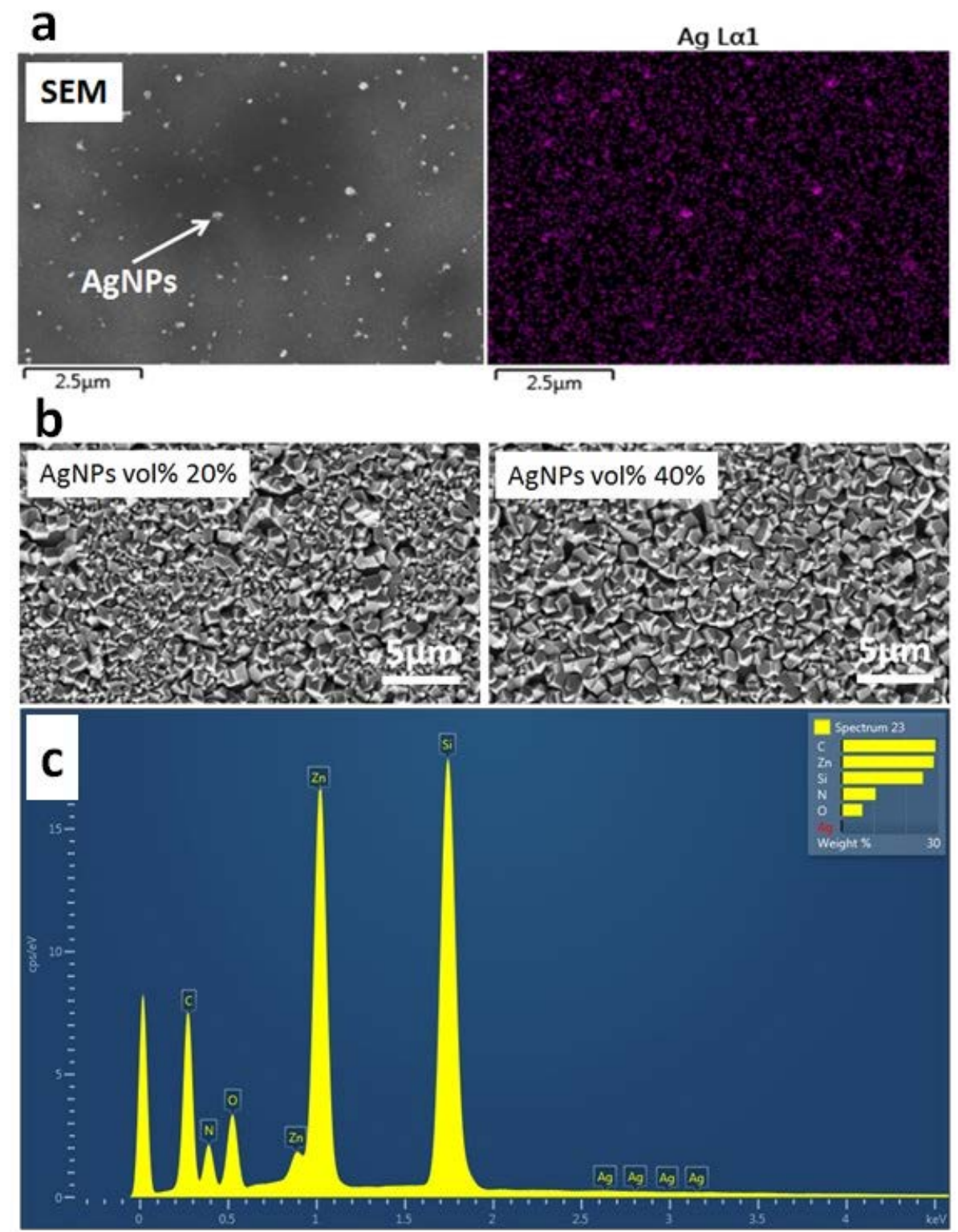

Figure S8. The effect of Ag NPs on the crystallization of ZIF-8 nanocrystals: (a) SEM image and Ag mapping of $\mathrm{ZnO}$ surface with dispensed $10 \mathrm{~nm}$ Ag NPs (20 vol. \%); (b) SEM image of substrate surface after solvothermal reaction; (c) EDX spectrum. 


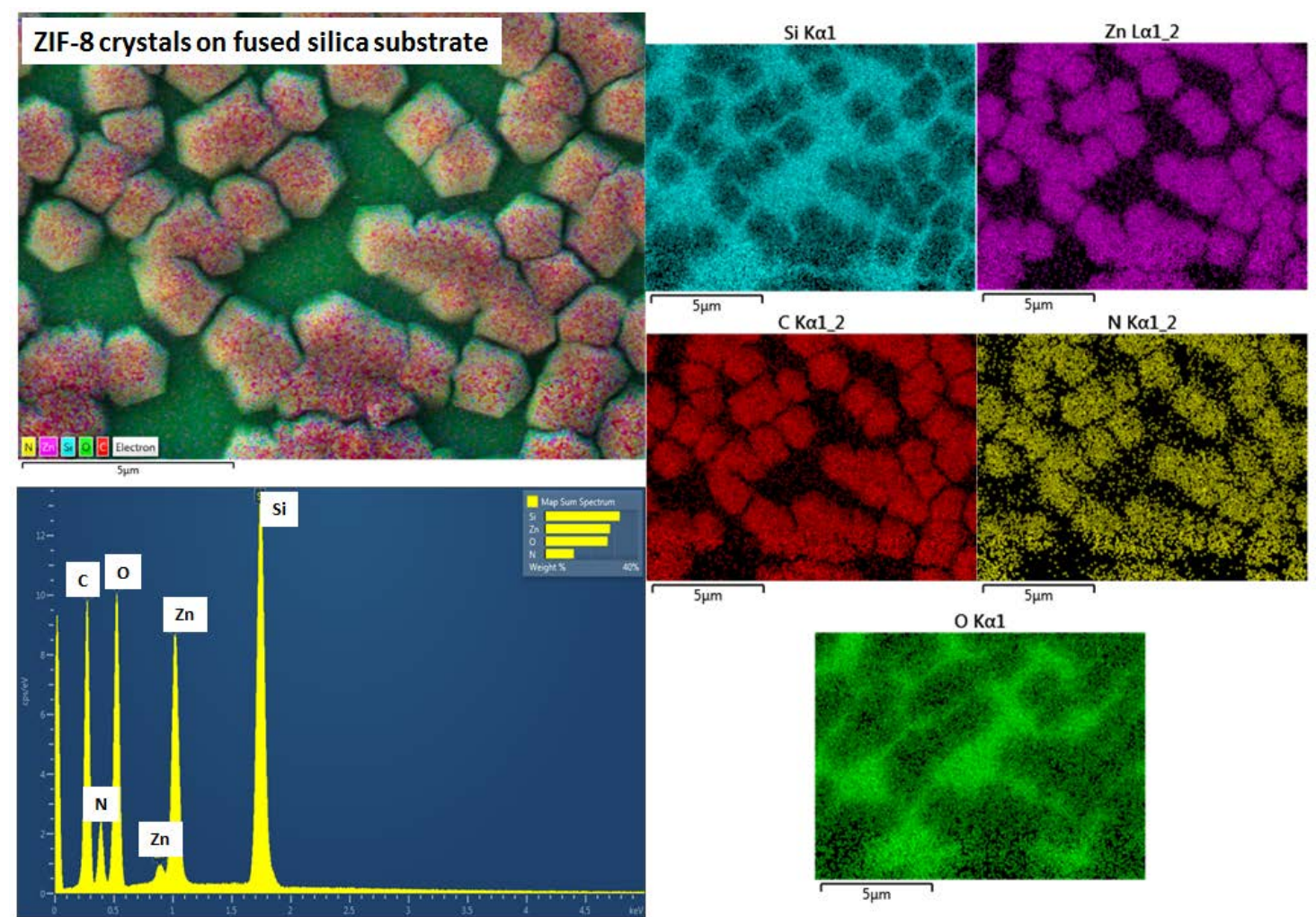

Figure S9. EDX elemental mapping and spectrum of isolated ZIF-8 crystals on fused silica substrate.

The concentration of added Au NPs is 60 vol. \%. 


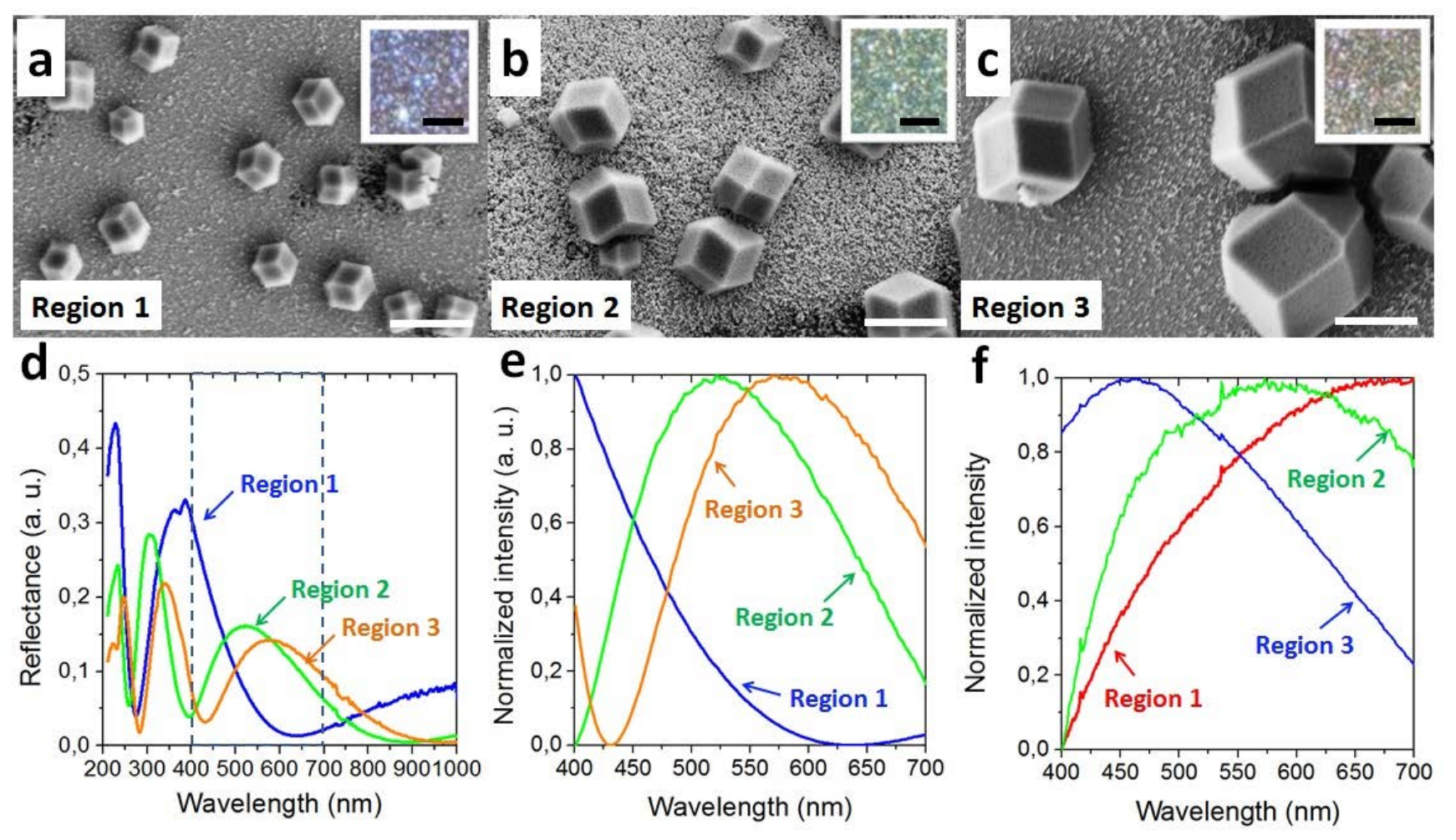

Figure S10. (a-c) SEM images of ZIF-8 nanocrystals with three different sizes (the scale bars are 1 $\mu \mathrm{m})$, the insets are corresponding images under dark field optical microscope (the scale bars are 10 $\mu m$ ); (d) Reflection spectra of three different regions measured by ellipsometry; (e) Reflection spectra in visible wavelengths; (e) Transmission spectra of three different regions measured by UV-visible spectroscopy. 

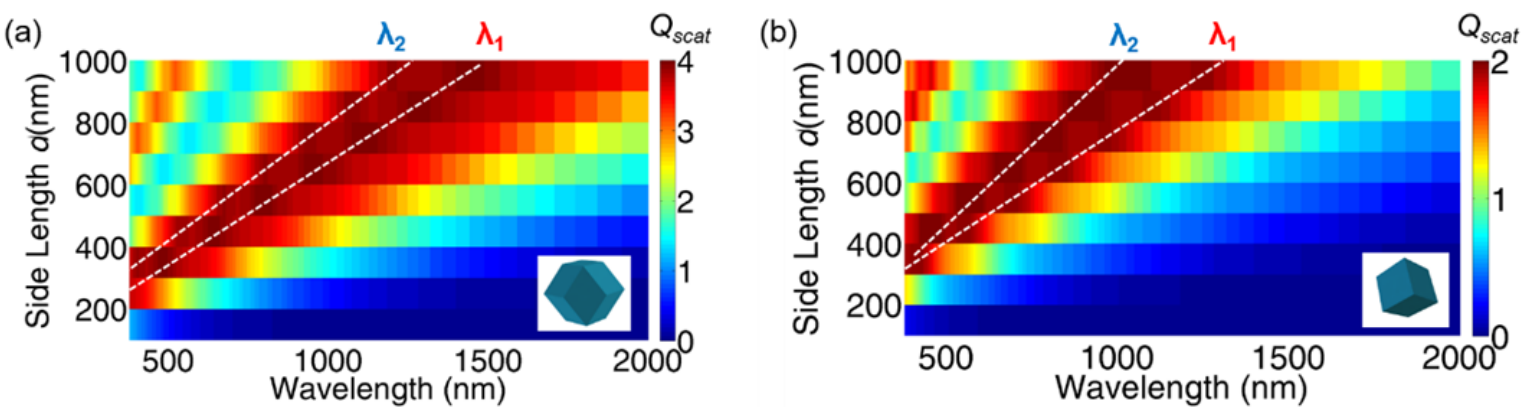

Figure S11. Two-dimensional map of the calculated scattering efficiency $Q_{\text {scat }}$ of ZIF-8 nanocrystals as a function of side length $d$ and incident wavelength. (a) is for rhombic dodecahedral while (b) is for cubic nanocrystals. 


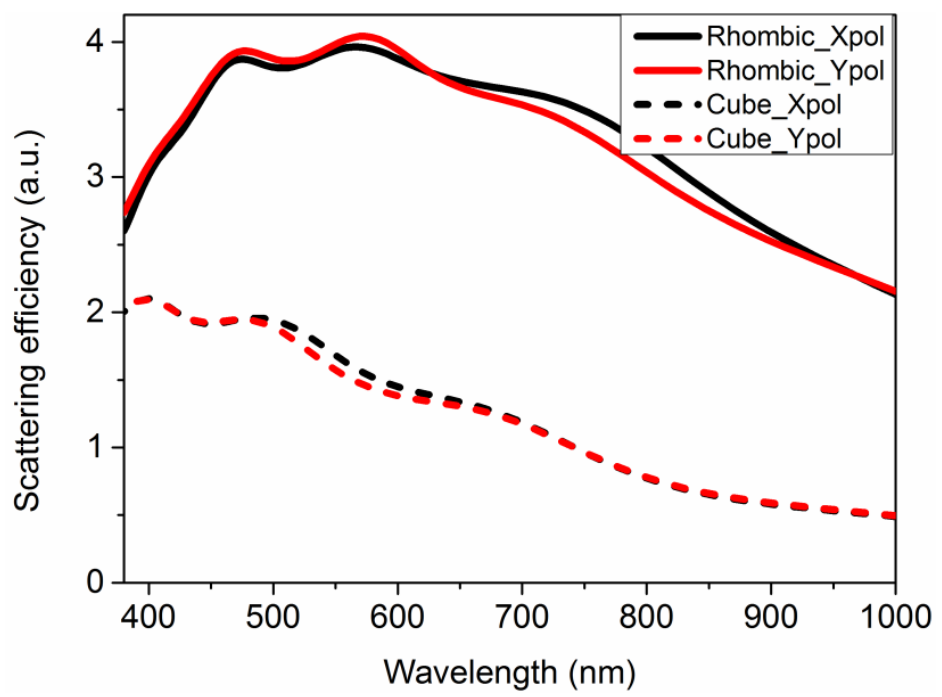

Figure S12. Calculated scattering efficiency $Q_{\text {scat }}$ of ZIF-8 nanocrystals with a fixed size $(d=350 \mathrm{~nm})$ but different morphologies and incident polarizations. The spectra show only a slight difference between two perpendicular incident polarizations due to the high-rotational symmetry of the crystal shape. 